\title{
Exploring Spatiotemporal Complexity of a Predator-Prey System with Migration and Diffusion by a Three-Chain Coupled Map Lattice
}

\author{
Tousheng Huang $(\mathbb{D}$, Huayong Zhang $\mathbb{D}$, Xuebing Cong $(\mathbb{D}$, Ge Pan $\mathbb{D}$, \\ Xiumin Zhang $\mathbb{B}$, and Zhao Liu $\mathbb{D}$ \\ Research Center for Engineering Ecology and Nonlinear Science, North China Electric Power University, Beijing 102206, China \\ Correspondence should be addressed to Huayong Zhang; rceens@ncepu.edu.cn
}

Received 12 January 2019; Accepted 3 March 2019; Published 5 May 2019

Academic Editor: Eric Campos-Canton

Copyright (c) 2019 Tousheng Huang et al. This is an open access article distributed under the Creative Commons Attribution License, which permits unrestricted use, distribution, and reproduction in any medium, provided the original work is properly cited.

\begin{abstract}
The topic of utilizing coupled map lattice to investigate complex spatiotemporal dynamics has attracted a lot of interest. For exploring the spatiotemporal complexity of a predator-prey system with migration and diffusion, a new three-chain coupled map lattice model is developed in this research. Based on Turing instability analysis, pattern formation conditions for the predator-prey system are derived. Via numerical simulation, rich Turing patterns are found with subtle self-organized structures under diffusiondriven and migration-driven mechanisms. With the variation of migration rates, the predator-prey system exhibits a gradual dynamical transition from diffusion-driven patterns to migration-driven patterns. Moreover, new results, the self-organization of non-Turing patterns, are also revealed. We find that even in the cases where the nonspatial predator-prey system reaches collapse, the migration can still drive pattern self-organization. These non-Turing patterns suggest many new possible ways for the coexistence of predator and prey in space, under the effects of migration and diffusion.
\end{abstract}

\section{Introduction}

Ecological systems are basically characterized by the interactions between populations and natural environment [1]. Among all of ecological interactions, predation is a significant type of interaction which occurs over a wide range of spatial and temporal domains $[2,3]$. Studies on predator-prey systems which have played an important role in ecological science can date back to the pioneering work of Lotka and Volterra in the early 1920 s $[4,5]$. Over the last one hundred years, rich results have been obtained on the complex dynamics of predator-prey systems $[6,7]$.

With the application of Turing's theory, the pattern formation of predator-prey systems has become a hot topic, attracting attention of ecologists and mathematical biologists [8-11]. For example, Yi et al. [12] investigated a diffusive predator-prey system with Holling type-II functional response and obtained spatiotemporal patterns which provide theoretical evidence to complex spatiotemporal dynamics, i.e., existence of loops of spatially nonhomogeneous periodic orbits and steady state solutions. Shi and Ruan [13], Song et al. [14], Song and Tang [15], and Cai et al. [16] explored Turing patterns in predator-prey systems, demonstrating that the system undergoing Turing-Hopf bifurcation can exhibit not only stationary Turing patterns but also temporal periodic patterns. Cai et al. [17] and Wang et al. [18] investigated Turing patterns as a result of disease spread, determining a special range for the disease parameters to control the diseases via studying the characteristics of the Turing patterns.

The pattern formation of predator-prey systems is always related to spatial movement of predator and prey populations [19]. Recently, research on mathematical biology has shown that diffusion and migration, the most two important spatial movements in nature, hold a crucial influence on spatiotemporal population dynamics [20]. For diffusion, it is based on the assumption that the motion of individuals of a given population is random and isotropic $[2,20]$. In the diffusion circumstance, Wang et al. [21] found complex dynamic patterns in a ratio-dependent predator-prey system, 
including chaos patterns and spiral waves which better explain the dynamics of aquatic communities in marine environment. Different from diffusion, migration means that the individuals can exhibit a correlated motion towards a certain direction instead of random motion $[2,20]$. This kind of motion may often lead to the self-organization of traveling spatial patterns in predator-prey systems [20].

In previous research on the pattern formation of predator-prey systems, investigations mainly focused on the cases with population diffusion [2]. Recently, the researchers have been interested in the spatial heterogeneities resulting from migration [20]. Sun et al. [2] revealed that migration can lead to transformation of Turing pattern into traveling pattern. Later, Liu [20] analyzed a predator-prey system with Holling-III type functional response combined with both diffusion and migration terms, detecting that the effects of migration on the wave behavior would lead to oscillation frequency increasing with wave number. Moreover, Zhang et al. [22] revealed that the combining effects of diffusion and migration can account for the dynamical complexity of predator-prey systems.

Mathematical modeling is one of the most effective methods to study the dynamics of predator-prey systems. As widely recognized, reaction-diffusion models have been well developed for revealing the predator-prey patterns $[2,3$, 20]. So far, most researchers have focused on the reactiondiffusion predator-prey models with continuous time and space. However, for the cases of patchy environment or fragmented habitat, discrete predator-prey models may be more reasonable and adequate [23, 24]. For example, Mistro et al. [23] considered that patchy and discontinuous environmental properties should be described more adequately by a spacediscrete model. They found that all typical invasion scenarios observed in continuous models can be observed as well in the discrete model and that very few artifacts of the regular lattice structure observed in the discrete model cannot be found in continuous models. Recently, a few researchers further developed a new type of spatially extended predator-prey model that is given by coupled map lattice (CML) $[23,24]$. It is found that the CMLs can describe rich spatiotemporal complexity and produce new attractive results of predatorprey systems [24-27].

A CML is characterized by discrete time, discrete space, and continuous states. In the 1980s, the CML was firstly developed to explore the spatiotemporal structure of coupled logistic map by Kaneko. Abundant spatiotemporal dynamics was revealed, including frozen chaos, spatiotemporal intermittency, defect turbulence, and fully developed spatiotemporal chaos [28-30]. Based on Kaneko's work, Mistro et al. [23], Rodrigues et al. [27], and Punithan et al. [31] proposed CML models for the reaction-diffusion predator-prey systems. Their research discovered new dynamical behaviors and provided reliable prediction for the predator-prey dynamics. Lately, Huang et al. [24, 25] further developed CML models through discretizing continuous reaction-diffusion model and detected a surprising variety of spatiotemporal patterns, demonstrating that the nonlinear mechanisms of CML can effectively capture the dynamical complexity of predator-prey systems $[31,32]$.
In this research, we intend to explore the spatiotemporal complexity of a predator-prey system with migration and diffusion by applying CML model. In such a system, there actually exist three distinctly different ecological processes: "reaction", diffusion, and migration. Based on previous thoughts for developing CML model $[30,33]$ and the ecological reality, we consider the three processes as separate stages. Corresponding to the three processes in the predatorprey system, a new three-chain CML model which holds ecological satisfaction can be developed. However, to the best of our knowledge, there is still scarce work which developed three-chain CML model to explore dynamical behaviors of the spatially extended predator-prey systems.

With the application of the three-chain CML model, new nonlinear characteristics of the predator-prey patterns may be found under the effects of both diffusion and migration. This work is organized as follows. In Section 2, we propose a three-chain CML model to describe the ratio-dependent predator-prey system with diffusion and migration and give the results of stability analysis. In Section 3, analysis of Turing instability of the predator-prey system is made to determine the pattern formation conditions. Section 4 provides numerical simulation results to demonstrate the spatiotemporal complexity. In Section 5, discussion and conclusions are described.

\section{Development of the Three-Chain Coupled Lattice Map Model}

In this research, we focus attention on the dynamics of a spatiotemporal ratio-dependent predator-prey system, which has received great attention among theoretical and mathematical biologists $[1,21]$. As described in literature, the governing equations of the nonspatial ratio-dependent predatorprey model take the following form [21]:

$$
\begin{aligned}
& \frac{d N}{d t}=r\left(1-\frac{N}{K}\right) N-\frac{\alpha N P}{P+\alpha h N}, \\
& \frac{d P}{d t}=\frac{\gamma \alpha N P}{P+\alpha h N}-\mu P,
\end{aligned}
$$

where $N$ and $P$ stand for prey and predator density, respectively; $r$ stands for maximal growth rate of the prey; $K$ describes the carrying capacity for the prey population; $\alpha$ is the capture rate of predator on prey; $h$ is the handling time; $\gamma$ is the conversion efficiency from prey to predator; $\mu$ is the predator mortality rate.

In order to reduce the number of parameters involved in the model, the equations in (1a) and (1b) are simplified via nondimensionalization. Here, taking

$$
\begin{aligned}
u & =\frac{\alpha h N}{\gamma K}, \\
v & =\frac{\alpha h P}{\gamma^{2} K}, \\
R & =\frac{r h}{\gamma},
\end{aligned}
$$




$$
\begin{aligned}
& Q=\frac{h \mu}{\gamma}, \\
& S=\frac{\alpha h}{\gamma}, \\
& \widehat{t}=\frac{\gamma t}{h},
\end{aligned}
$$

then we obtain the following simplified differential equations:

$$
\begin{aligned}
& \frac{d u}{d \widehat{t}}=R\left(1-\frac{u}{S}\right) u-\frac{S u v}{v+S u}, \\
& \frac{d v}{d \widehat{t}}=\frac{S u v}{v+S u}-Q v .
\end{aligned}
$$

The goal of the present study is to explore the spatiotemporal dynamics of the predator-prey system with migration and diffusion. Considering diffusion and migration of predator and prey populations based on (3a) and (3b), a spatiotemporal predator-prey model can be described as

$$
\begin{aligned}
& \frac{d u}{d \widehat{t}}+C_{1} \Gamma u=R\left(1-\frac{u}{S}\right) u-\frac{S u v}{v+S u}+D_{1} \nabla^{2} u, \\
& \frac{d v}{d t}+C_{2} \Gamma v=\frac{S u v}{v+S u}-Q v+D_{2} \nabla^{2} v,
\end{aligned}
$$

where $\nabla^{2}=\left(\partial^{2} / \partial x^{2}\right)+\left(\partial^{2} / \partial y^{2}\right)$ is the usual Laplacian operator in two-dimensional space; $\Gamma=\partial / \partial x$ is the advection operator, meaning that the individuals migrate along one direction, i.e., $x$ direction. One-directional migration of the populations is a common phenomenon in nature. For example, for the two-year-old hatchery-reared progeny of spawning brown trout in the case of the R. Imas, southwestern Norway, Jonsson et al. [34] found that migratory direction of juvenile fish and rivet fish population is always against and follows water current, respectively. $D_{1}$ and $D_{2}$ are prey and predator diffusion coefficients; $C_{1}$ and $C_{2}$ are the corresponding migration coefficients.

The CML model is then developed through discretizing system (4a) and (4b) [23]. At first, time interval and space interval for discretization are given. Generally, the dynamics of predator-prey system can be observed by a particular time scale, which can be defined by the generation span of predator and prey populations and measures the regeneration time of both populations. In this study, we denote the time scale on which predator-prey dynamics is observed as parameter $\tau$ and apply it as the time interval for discretization of system (4a) and (4b). The space interval used for discretization represents the space scale on which spatial movements of predator and prey take place. Its value can be defined by the maximum size of dwelling sites of predator and prey individuals. In this study, the space interval is denoted as parameter $l$.

Considering a two-dimensional rectangular lattice which includes $n \times n$ grid cells for establishing the CML model. The length of each grid cell is exactly the value of $l$. Each grid cell represents one site where predator and prey individuals dwell and is ascribed to two numbers, i.e., the prey density and the predator density. The prey and predator densities in each site change with time in course of the system dynamics, due to local inter- and intraspecific interactions as well as migration and diffusion between different sites [23]. Here, we use symbol $m$ ( $m \in N$, and $N$ describes the set of positive integers) to represent the time increasing with discrete iterations. With given initial time $t_{0}$, symbol $m$ means the $m$ th iteration and refers to the time $t_{0}+m \tau$. On this basis, two discrete state variables, $u_{(i, j, m)}$ and $v_{(i, j, m)}(i, j \in N)$, are defined, representing the prey density and the predator density in the $(i, j)$ site at iteration $m$ (for simplification we also use time $m$ to refer to the time $t_{0}+m \tau$ ).

At each discrete step from iteration $m$ to $m+1$, the dynamics of predator and prey consists of three parts, reaction, diffusion, and migration. In literature, when CML is applied to study the predator-prey systems, the "reaction" between predator and prey and the diffusion of both populations are often regarded as two distinctly different stages [23, 27, 31]. Hassell et al. [35] argued that with such processes of segregating and corresponding operators splitting, counterintuitive results, such as the production of negative local population densities at some extreme cases [35], can be avoided. Moreover, Hassell et al. suggested that the processes of segregating and operators splitting are actually more biologically sensible. Mathematically, splitting of diffusion terms and advection terms in an advection-diffusion partial differential equation is a canonical example of operator splitting [36]. It is faster to compute the solution of the splitting terms separately than to compute the solution directly when they are treated together [33]. Based on previous research on the CML, we further consider the migration of populations as another different stage in this research, split the terms of reaction, diffusion, and migration, and solve them separately.

Such consideration of treating reaction, diffusion, and migration processes as three separate stages is based on following ecological facts. First, migration and diffusion may often take place when the populations and individuals seek for new habitats and necessary life conditions, whereas the reaction process mainly occurs when the populations settle down in stable habitats. Therefore, the reaction stage can be considered as a separate stage different from diffusion and migration. Second, migration and diffusion represent directional and unidirectional population motions which show difference in spatial scale. The population migration often takes place in a huge spatial scale; e.g., the migration of fish populations may cross over thousand miles, whereas the population diffusion may merely take place in the spatial scale of a local habitat. Third, the population migration and diffusion may occur in different stages; specifically, phenomenon where populations migrate first and then randomly spread indeed exists in nature. In the case of large herbivores, the population often migrates between discrete home ranges as a means of enhancing access to high quality food and/or reducing the risk of predation [37]. After settling down in new habitats, individuals of the population move randomly in the home range to acquire enough food. Simultaneously, since the predator is dependent on the prey to survive, the motion of the predator population that feeds on the herbivores also 
follows the same way. Based on the above descriptions, it is reasonable to consider the processes of reaction, diffusion, and migration as separate stages. Moreover, the sequence of the three stages can be considered as migration, diffusion, and reaction, respectively.

Therefore, in the CML of predator-prey systems with migration and diffusion, three parts of population dynamics are modeled as distinctly different stages, (a) the migration stage, (b) the diffusion stage, and (c) the "reaction" stage. The first stage is obtained by discretizing the population migrating dynamics with time interval $\tau$ and space interval $l$. Then we obtain

$$
\begin{aligned}
& u_{(i, j, m)}^{\prime}=u_{(i, j, m)}+\frac{\tau}{l} C_{1} \Gamma_{\mathrm{d}} u_{(i, j, m)}, \\
& v_{(i, j, m)}^{\prime}=v_{(i, j, m)}+\frac{\tau}{l} C_{2} \Gamma_{\mathrm{d}} v_{(i, j, m)},
\end{aligned}
$$

where $\Gamma_{d}$ is the discrete advection operator, i.e.,

$$
\Gamma_{\mathrm{d}} \psi_{(i, j, m)}=\psi_{(i-1, j, m)}-\psi_{(i, j, m)},
$$

where $\psi$ represents the variables of $u$ and $v$. Likewise, the diffusion stage can be described by the following equations:

$$
\begin{aligned}
& u_{(i, j, m)}^{\prime \prime}=u_{(i, j, m)}^{\prime}+\frac{\tau}{l^{2}} D_{1} \Delta_{\mathrm{d}} u_{(i, j, m)}^{\prime}, \\
& v_{(i, j, m)}^{\prime \prime}=v_{(i, j, m)}^{\prime}+\frac{\tau}{l^{2}} D_{2} \Delta_{\mathrm{d}} v_{(i, j, m)}^{\prime},
\end{aligned}
$$

where $\Delta_{\mathrm{d}}$ is the discrete Laplacian operator, i.e.,

$$
\begin{aligned}
\Delta_{\mathrm{d}} \psi_{(i, j, m)}= & \psi_{(i+1, j, m)}+\psi_{(i-1, j, m)}+\psi_{(i, j+1, m)} \\
& +\psi_{(i, j-1, m)}-4 \psi_{(i, j, m)} .
\end{aligned}
$$

For the last "reaction" stage, we discretize the nonspatial system (3a) and (3b) and obtain

$$
\begin{aligned}
& u_{(i, j, m+1)}=f_{1}\left(u_{(i, j, m)}^{\prime \prime}, v_{(i, j, m)}^{\prime \prime}\right), \\
& v_{(i, j, m+1)}=g_{1}\left(u_{(i, j, m)}^{\prime \prime}, v_{(i, j, m)}^{\prime \prime}\right),
\end{aligned}
$$

in which

$$
\begin{aligned}
& f_{1}(u, v)=u+\tau\left(R\left(1-\frac{u}{S}\right) u-\frac{S u v}{v+S u}\right), \\
& g_{1}(u, v)=v+\tau\left(\frac{S u v}{v+S u}-Q v\right) .
\end{aligned}
$$

Equations (5a), (5b), (5c), (5d), (5e), (5f), (5g), (5h), (5i), and (5j) give the expression of the three-chain CML model which describes a spatiotemporally discrete ratiodependent type predator-prey system with migration and diffusion. Such a CML can be viewed as a discretization of the continuous reaction-advection-diffusion predatorprey model. In the migration stage, the predator and prey individuals move from cell $(i-1, j)$ to cell $(i, j)$, i.e., along the negative $x$ direction, and the corresponding migration rates are $C_{1}$ and $C_{2}$, respectively. This stage describes the process that populations migrate between different habitats.
Then the system enters the diffusion stage; the predator and prey individuals disperse to four adjacent cells with diffusion rates as $D_{1}$ and $D_{2}$, demonstrating predator and prey moving randomly in local habitat which may be fragmented. In the last stage, the predator and prey populations react under the predation relation described by $(5 i)$ and $(5 j)$. From the point view of ecological significance, the CML model can be regarded as an alternative to the corresponding continuous reaction-advection-diffusion model. All the parameters used in the CML should be positive. Moreover, for ensuring nonnegativity of $u$ and $v$ and convergence of the CML, the parameter values should be provided to make $\tau \mathrm{C}_{i} / l$ and $\tau D_{i} / l^{2}$ $(i=1,2)$ less than $0.5[26]$.

In order to explore the spatiotemporal complexity of the discrete predator-prey system, firstly the nonspatial dynamics of system (5a), (5b), (5c), (5d), (5e), (5f), (5g), (5h), (5i), and (5j) should be investigated. Let $C_{i}$ and $D_{i}(i=1,2)$ be zero; the equations of the CML model become

$$
\begin{aligned}
& u_{m+1}=u_{m}+\tau\left(R\left(1-\frac{u_{m}}{S}\right) u_{m}-\frac{S u_{m} v_{m}}{v_{m}+S u_{m}}\right), \\
& v_{m+1}=v_{m}+\tau\left(\frac{S u_{m} v_{m}}{v_{m}+S u_{m}}-Q v_{m}\right) .
\end{aligned}
$$

It should be noticed that the dynamical behaviors of (6a) and (6b) represent the homogeneous states of the spatiotemporally discrete predator-prey system. One of the important dynamical behaviors is the fixed point, which represents the homogeneous stationary state of the system. Via the definition of fixed point of difference equations, two fixed points of (6a) and (6b) can be obtained as follows:

$$
\begin{aligned}
& \left(u_{1}, v_{1}\right)=(S, 0), \\
& \left(u_{2}, v_{2}\right) \\
& =\left(\frac{S(R+S(Q-1))}{R}, \frac{S^{2}(1-Q)(R+S(Q-1))}{Q R}\right), \\
& 1-\frac{R}{S}<Q<1 .
\end{aligned}
$$

$\left(u_{1}, v_{1}\right)$ means the extinction of the predator, and system (6a) and (6b) will finally converge to the state with prey reaching carrying capacity. $\left(u_{2}, v_{2}\right)$ describes the coexistence of the predator and prey. The stability of this fixed point indicates whether predator and prey can stably coexist. To determine the stability of $\left(u_{2}, v_{2}\right)$, Jacobian matrix is applied. The Jacobian matrix associated with system (6a) and (6b) at any point is calculated as

$$
\begin{aligned}
& J(u, v) \\
& =\left(\begin{array}{cc}
1+\tau\left(R-\frac{2 R u}{S}-\frac{S v^{2}}{(v+S u)^{2}}\right) & \frac{-\tau S^{2} u^{2}}{(v+S u)^{2}} \\
\frac{\tau S v^{2}}{(v+S u)^{2}} & 1+\tau\left(\frac{S^{2} u^{2}}{(v+S u)^{2}}-Q\right)
\end{array}\right) .
\end{aligned}
$$

Substituting the value of fixed points into Jacobian matrix (8) and then calculating the eigenvalues of the matrix, from 
the values of the two eigenvalues, the stability of the two fixed points is determined. If the modules of the two eigenvalues are both smaller than one, the corresponding fixed point is stable; if the modulus of one of the eigenvalues is larger than one, the corresponding fixed point is unstable. After calculation, the two eigenvalues of $\left(u_{1}, v_{1}\right)$ are $1-\tau R$ and $1+\tau(1-$ $Q)$. In the case where positive $\left(u_{2}, v_{2}\right)$ exists, the condition $1-R / S<Q<1$ must hold. Therefore, we have $1+\tau(1-Q)>1$, and the fixed point $\left(u_{1}, v_{1}\right)$ is unstable. The two eigenvalues of $\left(u_{2}, v_{2}\right)$ are found as

$$
\lambda_{1,2}=\frac{-p}{2} \pm \frac{\sqrt{p^{2}-4 q}}{2}
$$

where

$$
\begin{aligned}
p= & -2+\tau R-\tau(Q-1)(Q-S(Q+1)) \\
q= & 1-\tau(\tau Q(Q-1)+1) R-\tau^{2} S Q(Q-1)^{2} \\
& +\tau(Q-1)(Q-S(Q+1)) .
\end{aligned}
$$

According to the above determination, we find that $\left(u_{2}, v_{2}\right)$ is stable if

$$
\begin{aligned}
& -p<1+q, \\
& -p>-1-q,
\end{aligned}
$$

$$
q<1
$$

The stability of homogeneous stationary state reveals whether the system can resist external disturbances and stay on the stable state. The above stability analysis suggests that the predator-prey system may reach stable homogeneous stationary state. When the conditions in (11) are satisfied, the predator and prey populations in the system can stably coexist and the system will eventually evolve to a state where the predator and prey densities remain at $u_{2}$ and $v_{2}$ in entire space and do not change with time. Even when homogeneous perturbations exert influences, the system will turn back to this state. However, when the conditions in (11) are not in satisfaction, the predator-prey system will collapse at the end; i.e., the predator population will be extinct and the prey population will grow to its carrying capacity.

\section{Turing Instability Analysis}

In the three-chain CML developed above, the combination effects of diffusion and migration may destabilize a spatially homogeneous stationary state of the predator-prey system that would be stable in the absence of population motions in space, i.e., triggering the Turing instability [38], which leads to spatial pattern self-organization in the discrete predatorprey system.

To perform Turing instability analysis, spatially heterogeneous perturbations are introduced to linearize the discrete predator-prey system around $\left(u_{2}, v_{2}\right)$. We assume

$$
\begin{aligned}
& u_{(i, j, m)}=u_{2}+\widetilde{u}_{(i, j, m)}, \\
& v_{(i, j, m)}=v_{2}+\widetilde{v}_{(i, j, m)},
\end{aligned}
$$

where $\widetilde{u}_{(i, j, m)} \ll u_{2}$ and $\widetilde{v}_{(i, j, m)} \ll v_{2}$. Substituting (12) into (5a), (5b), (5c), (5d), (5e), (5f), (5g), (5h), (5i), and (5j) directly yields

$$
\begin{aligned}
& u_{(i, j, m)}^{\prime}=u_{2}+\widetilde{u}_{(i, j, m)}+\frac{\tau}{l} C_{1} \Gamma_{\mathrm{d}} \widetilde{u}_{(i, j, m)}, \\
& v_{(i, j, m)}^{\prime}=v_{2}+\widetilde{v}_{(i, j, m)}+\frac{\tau}{l} C_{2} \Gamma_{\mathrm{d}} \widetilde{v}_{(i, j, m)},
\end{aligned}
$$

$$
u_{(i, j, m)}^{\prime \prime}=u_{(i, j, m)}^{\prime}+\frac{\tau}{l^{2}} D_{1} \Delta_{\mathrm{d}} \tilde{u}_{(i, j, m)}
$$$$
+\frac{\tau^{2}}{l^{3}} C_{1} D_{1} \Delta_{\mathrm{d}} \Gamma_{d} \widetilde{u}_{(i, j, m)},
$$

$$
v_{(i, j, m)}^{\prime \prime}=v_{(i, j, m)}^{\prime}+\frac{\tau}{l^{2}} D_{2} \Delta_{\mathrm{d}} \widetilde{v}_{(i, j, m)}
$$

$$
+\frac{\tau^{2}}{l^{3}} C_{2} D_{2} \Delta_{\mathrm{d}} \Gamma_{d} \widetilde{v}_{(i, j, m)}
$$

$$
\begin{aligned}
& u_{2}+\widetilde{u}_{(i, j, m+1)}=f_{1}\left(u_{(i, j, m)}^{\prime \prime}, v_{(i, j, m)}^{\prime \prime}\right), \\
& v_{2}+\widetilde{v}_{(i, j, m+1)}=g_{1}\left(u_{(i, j, m)}^{\prime \prime}, v_{(i, j, m)}^{\prime \prime}\right) .
\end{aligned}
$$

Linearizing (13e) and (13f) around $\left(u_{2}, v_{2}\right)$, we obtain

$$
\begin{aligned}
u_{2}+\widetilde{u}_{(i, j, m+1)}= & f_{1}\left(u_{2}, v_{2}\right)+a_{11}\left(u_{(i, j, m)}^{\prime \prime}-u_{2}\right) \\
& +a_{12}\left(v_{(i, j, m)}^{\prime \prime}-v_{2}\right), \\
v_{2}+\widetilde{v}_{(i, j, m+1)}= & g_{1}\left(u_{2}, v_{2}\right)+a_{21}\left(u_{(i, j, m)}^{\prime \prime}-u_{2}\right) \\
& +a_{22}\left(v_{(i, j, m)}^{\prime \prime}-v_{2}\right),
\end{aligned}
$$

where

$$
\begin{aligned}
& a_{11}=\left.\frac{\partial f_{1}}{\partial u}\right|_{\left(u_{2}, v_{2}\right)}, \\
& a_{12}=\left.\frac{\partial f_{1}}{\partial v}\right|_{\left(u_{2}, v_{2}\right)}, \\
& a_{21}=\left.\frac{\partial g_{1}}{\partial u}\right|_{\left(u_{2}, v_{2}\right)}, \\
& a_{22}=\left.\frac{\partial g_{1}}{\partial v}\right|_{\left(u_{2}, v_{2}\right)} .
\end{aligned}
$$


Simplifying (14a) and (14b) by applying (13a)-(13d) leads to

$$
\left(\begin{array}{c}
\widetilde{u}_{(i, j, m+1)} \\
\widetilde{v}_{(i, j, m+1)}
\end{array}\right)=\left(\begin{array}{cc}
a_{11} & a_{12} \\
a_{21} & a_{22}
\end{array}\right)\left(\begin{array}{c}
\widetilde{u}_{(i, j, m)}+\frac{\tau}{l} C_{1} \Gamma_{\mathrm{d}} \widetilde{u}_{(i, j, m)}+\frac{\tau}{l^{2}} D_{1} \Delta_{\mathrm{d}} \widetilde{u}_{(i, j, m)}+\frac{\tau^{2}}{l^{3}} C_{1} D_{1} \Delta_{\mathrm{d}} \Gamma_{\mathrm{d}} \widetilde{u}_{(i, j, m)} \\
\widetilde{v}_{(i, j, m)}+\frac{\tau}{l} C_{2} \Gamma_{\mathrm{d}} \widetilde{v}_{(i, j, m)}+\frac{\tau}{l^{2}} D_{2} \Delta_{\mathrm{d}} \widetilde{v}_{(i, j, m)}+\frac{\tau^{2}}{l^{3}} C_{2} D_{2} \Delta_{\mathrm{d}} \Gamma_{\mathrm{d}} \widetilde{v}_{(i, j, m)}
\end{array}\right),
$$

which is equivalent to

$$
\begin{aligned}
& \widetilde{u}_{(i, j, m+1)}=a_{11} \widetilde{u}_{(i, j, m)}+a_{12} \widetilde{v}_{(i, j, m)} \\
& +\frac{\tau^{2}}{l^{3}}\left(a_{11} C_{1} D_{1} \Delta_{\mathrm{d}} \Gamma_{\mathrm{d}} \widetilde{u}_{(i, j, m)}\right. \\
& \left.+a_{12} C_{2} D_{2} \Delta_{\mathrm{d}} \Gamma_{\mathrm{d}} \widetilde{v}_{(i, j, m)}\right)+\frac{\tau}{l^{2}}\left(a_{11} D_{1} \Delta_{\mathrm{d}} \widetilde{u}_{(i, j, m)}\right. \\
& \left.+a_{12} D_{2} \Delta_{\mathrm{d}} \widetilde{v}_{(i, j, m)}\right)+\frac{\tau}{l}\left(a_{11} C_{1} \Gamma_{\mathrm{d}} \widetilde{u}_{(i, j, m)}\right. \\
& \left.+a_{12} C_{2} \Gamma_{\mathrm{d}} \widetilde{v}_{(i, j, m)}\right), \\
& \widetilde{v}_{(i, j, m+1)}=a_{21} \widetilde{u}_{(i, j, m)}+a_{22} \widetilde{v}_{(i, j, m)} \\
& +\frac{\tau^{2}}{l^{3}}\left(a_{21} C_{1} D_{1} \Delta_{\mathrm{d}} \Gamma_{\mathrm{d}} \widetilde{u}_{(i, j, m)}\right. \\
& \left.+a_{22} C_{2} D_{2} \Delta_{\mathrm{d}} \Gamma_{\mathrm{d}} \widetilde{v}_{(i, j, m)}\right)+\frac{\tau}{l^{2}}\left(a_{21} D_{1} \Delta_{\mathrm{d}} \widetilde{u}_{(i, j, m)}\right. \\
& \left.+a_{22} D_{2} \Delta_{\mathrm{d}} \widetilde{v}_{(i, j, m)}\right)+\frac{\tau}{l}\left(a_{21} C_{1} \Gamma_{\mathrm{d}} \widetilde{u}_{(i, j, m)}\right. \\
& \left.+a_{22} C_{2} \Gamma_{\mathrm{d}} \widetilde{v}_{(i, j, m)}\right) .
\end{aligned}
$$

Since

$$
\begin{aligned}
\Gamma_{\mathrm{d}}\left(\Delta_{\mathrm{d}} u_{(i, j, m)}\right)= & \Delta_{\mathrm{d}}\left(\Gamma_{\mathrm{d}} u_{(i, j, m)}\right) \\
= & 5 u_{(i, j, m)}-5 u_{(i-1, j, m)}-u_{(i+1, j, m)} \\
& +u_{(i-2, j, m)}+u_{(i-1, j+1, m)}-u_{(i, j+1, m)} \\
& +u_{(i-1, j-1, m)}-u_{(i, j-1, m)},
\end{aligned}
$$

the discrete operators $\Gamma_{\mathrm{d}}$ and $\Delta_{\mathrm{d}}$ are commuting operators. Let $\Lambda_{\mathrm{d}}=\Delta_{\mathrm{d}} \Gamma_{\mathrm{d}}$, and we can verify that $\Gamma_{\mathrm{d}}, \Delta_{\mathrm{d}}$, and $\Lambda_{\mathrm{d}}$ are also commuting, i.e.,

$$
\begin{aligned}
\Gamma_{\mathrm{d}} \Lambda_{\mathrm{d}} & =\nabla_{d}\left(\Delta_{\mathrm{d}} \Gamma_{\mathrm{d}}\right)=\Gamma_{\mathrm{d}} \Delta_{\mathrm{d}} \Gamma_{\mathrm{d}}=\left(\Gamma_{\mathrm{d}} \Delta_{\mathrm{d}}\right) \Gamma_{\mathrm{d}}=\Lambda_{\mathrm{d}} \Gamma_{\mathrm{d}} \\
\Delta_{\mathrm{d}} \Lambda_{\mathrm{d}} & =\Delta_{\mathrm{d}}\left(\Gamma_{\mathrm{d}} \Delta_{\mathrm{d}}\right)=\Delta_{\mathrm{d}} \Gamma_{\mathrm{d}} \Delta_{\mathrm{d}}=\left(\Delta_{\mathrm{d}} \Gamma_{\mathrm{d}}\right) \Delta_{\mathrm{d}} \\
& =\Lambda_{\mathrm{d}} \Delta_{\mathrm{d}}
\end{aligned}
$$

Hence, the three operators have a group of common eigenfunctions. Moreover, to determine the eigenvalues of $\Gamma_{d}, \Delta_{d}$, and $\Lambda_{\mathrm{d}}$, we consider the following equations:

$$
\begin{gathered}
\Gamma_{\mathrm{d}} X_{\overline{i j}}+\lambda_{1 \overline{i j}}^{\prime} X_{\overline{i j}}=0, \\
\Delta_{\mathrm{d}} X_{\overline{i j}}+\lambda_{2 \overline{i j}}^{\prime} X_{\overline{i j}}=0, \\
\Lambda_{\mathrm{d}} X_{\overline{i j}}+\lambda_{3 \overline{i j}}^{\prime} X_{\overline{i j}}=0,
\end{gathered}
$$

with periodic boundary condition. As per the method described in Bai and Zhang [39], the eigenvalues of the three operators are solved and described as

$$
\begin{aligned}
& \lambda_{1 \overline{i j}}^{\prime}=2 \sin \frac{(\bar{i}-1) \pi}{n} \exp \left\{\hat{i}\left(\frac{\pi}{2}-\frac{(\bar{i}-1) \pi}{n}\right)\right\}, \\
& \lambda_{2 \overline{i j}}^{\prime}=4\left(\sin ^{2} \frac{(\bar{i}-1) \pi}{n}+\sin ^{2} \frac{(\bar{j}-1) \pi}{n}\right) . \\
& \lambda_{3 \overline{i j}}^{\prime}=-8\left(\sin ^{3} \frac{(\bar{i}-1) \pi}{n}\right. \\
&\left.\quad+\sin \frac{(\bar{i}-1) \pi}{n} \sin ^{2} \frac{(\bar{j}-1) \pi}{n}\right) \\
& \quad \cdot \exp \left\{\hat{i}\left(\frac{\pi}{2}-\frac{(\bar{i}-1) \pi}{n}\right)\right\},
\end{aligned}
$$

where the symbols $\bar{i}$ and $\bar{j}$ represent wavenumbers, $\bar{i}, \bar{j} \in$ $\{1,2,3, \cdots, n\}$, and $\hat{i}=\sqrt{-1}$.

Assuming that $X_{\overline{i j}}^{i j}$ is the common eigenfunction corresponding to the eigenvalues $\lambda_{1 \bar{i} \bar{j}}^{\prime}, \lambda_{2 \bar{i} \bar{j}}^{\prime}$, and $\lambda_{3 \overline{i j}}^{\prime}$ and using $X_{\overline{i j}}^{i j}$ to multiply (17a) and (17b), we obtain

$$
\begin{aligned}
& X_{\overline{i j}}^{i j} \widetilde{u}_{(i, j, m+1)}=a_{11} X_{\overline{i j}}^{i j} \widetilde{u}_{(i, j, m)}+a_{12} X_{\overline{i j}}^{i j} \widetilde{v}_{(i, j, m)} \\
& +\frac{\tau}{l}\left(a_{11} C_{1} X_{i j}^{i j} \Gamma_{\mathrm{d}} \widetilde{u}_{(i, j, m)}+a_{12} C_{2} X_{\overline{i j}}^{i j} \Gamma_{\mathrm{d}} \widetilde{v}_{(i, j, m)}\right) \\
& +\frac{\tau}{l^{2}}\left(a_{11} D_{1} X_{\overline{i j}}^{i j} \Delta_{\mathrm{d}} \widetilde{u}_{(i, j, m)}+a_{12} D_{2} X_{\overline{i j}}^{i j} \Delta_{\mathrm{d}} \widetilde{v}_{(i, j, m)}\right) \\
& +\frac{\tau^{2}}{l^{3}}\left(a_{11} C_{1} D_{1} X_{\overline{i j}}^{i j} \Lambda_{\mathrm{d}} \widetilde{u}_{(i, j, m)}\right. \\
& \left.+a_{12} C_{2} D_{2} X_{\overline{i j}}^{i j} \Lambda_{\mathrm{d}} \widetilde{v}_{(i, j, m)}\right)
\end{aligned}
$$




$$
\begin{aligned}
& X_{\overline{i j}}^{i j} \widetilde{v}_{(i, j, m+1)}=a_{21} X_{i j}^{i j} \widetilde{u}_{(i, j, m)}+a_{22} X_{i j}^{i j} \widetilde{v}_{(i, j, m)} \\
& \quad+\frac{\tau}{l}\left(a_{21} C_{1} X_{i j}^{i j} \Gamma_{\mathrm{d}} \widetilde{u}_{(i, j, m)}+a_{22} C_{2} X_{i j}^{i j} \Gamma_{\mathrm{d}} \widetilde{v}_{(i, j, m)}\right) \\
& \quad+\frac{\tau}{l^{2}}\left(a_{21} D_{1} X_{i j}^{i j} \Delta_{\mathrm{d}} \widetilde{u}_{(i, j, m)}+a_{22} D_{2} X_{i j}^{i j} \Delta_{\mathrm{d}} \widetilde{v}_{(i, j, m)}\right) \\
& \quad+\frac{\tau^{2}}{l^{3}}\left(a_{21} C_{1} D_{1} X_{i j}^{i j} \Lambda_{\mathrm{d}} \widetilde{u}_{(i, j, m)}\right. \\
& \left.\quad+a_{22} C_{2} D_{2} X_{i j}^{i j} \Lambda_{\mathrm{d}} \widetilde{v}_{(i, j, m)}\right) .
\end{aligned}
$$

Summing (24a) and (24b) for all of $i$ and $j$ and letting

$$
\begin{aligned}
& \bar{u}_{m}=\sum_{i, j=1}^{n} X_{\overline{i j}}^{i j} \widetilde{\mathcal{u}}_{(i, j, m)}, \\
& \bar{v}_{m}=\sum_{i, j=1}^{n} X_{\overline{i j}}^{i j} \widetilde{v}_{(i, j, m)},
\end{aligned}
$$

then we have

$$
\begin{aligned}
\bar{u}_{m+1} & =a_{11}\left(1-\frac{\tau C_{1} \lambda_{1 \bar{i}}^{\prime}}{l}-\frac{\tau D_{1} \lambda_{2 \overline{i j}}^{\prime}}{l^{2}}\right. \\
+ & \left.\frac{\tau^{2} C_{1} D_{1} \lambda_{1 \bar{j} j}^{\prime} \lambda_{2 \bar{i} j}^{\prime}}{l^{3}}\right) \bar{u}_{m}+a_{12}\left(1-\frac{\tau C_{2} \lambda_{1 \overline{i j}}^{\prime}}{l}\right.
\end{aligned}
$$

$$
\begin{aligned}
& \bar{J}=\left(\begin{array}{ll}
A_{11} & A_{12} \\
A_{21} & A_{22}
\end{array}\right)
\end{aligned}
$$

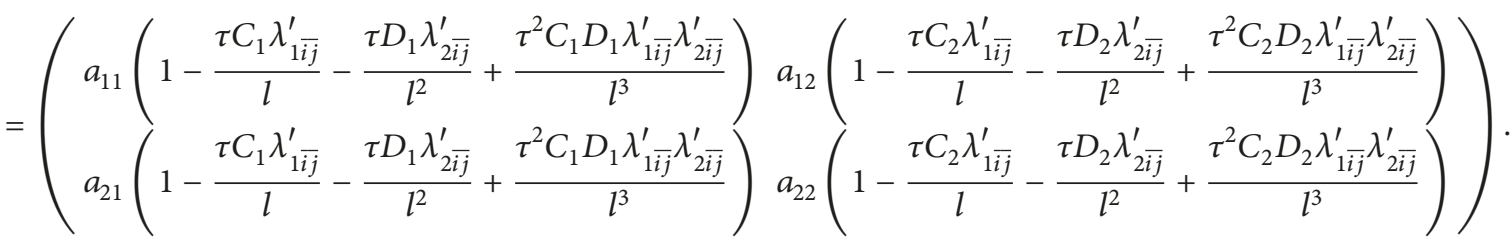

$$
\begin{gathered}
\left.-\frac{\tau D_{2} \lambda_{2 \overline{i j}}^{\prime}}{l^{2}}+\frac{\tau^{2} C_{2} D_{2} \lambda_{1 \overline{i j}}^{\prime} \lambda_{2 i \bar{j}}^{\prime}}{l^{3}}\right) \bar{v}_{m}, \\
\bar{v}_{m+1}=a_{21}\left(1-\frac{\tau C_{1} \lambda_{1 \overline{i j}}^{\prime}}{l}-\frac{\tau D_{1} \lambda_{2 \bar{i}}^{\prime}}{l^{2}}\right. \\
\left.+\frac{\tau^{2} C_{1} D_{1} \lambda_{1 \bar{i} j}^{\prime} \lambda_{2 i \bar{j}}^{\prime}}{l^{3}}\right) \bar{u}_{m}+a_{22}\left(1-\frac{\tau C_{2} \lambda_{1 \overline{i j}}^{\prime}}{l}\right. \\
\left.-\frac{\tau D_{2} \lambda_{2 i \bar{j}}^{\prime}}{l^{2}}+\frac{\tau^{2} C_{2} D_{2} \lambda_{1 \overline{i j}}^{\prime} \lambda_{2 i \bar{j}}^{\prime}}{l^{3}}\right) \bar{v}_{m} .
\end{gathered}
$$

Rewrite (24a) and (24b) in the following form:

$$
\left(\begin{array}{c}
\bar{u}_{m+1} \\
\bar{v}_{m+1}
\end{array}\right)=\bar{J}\left(\begin{array}{c}
\bar{u}_{m} \\
\bar{v}_{m}
\end{array}\right),
$$

in which
The two eigenvalues of the system (25) are determined as

$$
\begin{aligned}
& \bar{\lambda}_{ \pm} \\
& \quad=\frac{1}{2}\left(\left(A_{11}+A_{12}\right) \pm \sqrt{\left(A_{11}-A_{12}\right)^{2}+4 A_{12} A_{21}}\right) .
\end{aligned}
$$

Since (25) describes the dynamics of spatially heterogeneous perturbations integrating all the sites, when (25) converges, the discrete predator-prey system will go back to $\left(u_{2}, v_{2}\right)$, whereas when divergence of (27) occurs, Turing instability occurs and lead to the self-organization of Turing patterns. Hence, the occurrence conditions for Turing instability can be described as $[25,26,32]$

$$
L=\max _{\bar{i}, \bar{j}=1}^{n}\left\{\max \left(\left|\bar{\lambda}_{+}\right|,\left|\bar{\lambda}_{-}\right|\right)\right\}>1 .
$$

\section{Numerical Simulations}

The theoretical results given in previous sections reveal that the spatiotemporally discrete predator-prey system can produce Turing patterns with parameter values satisfying conditions (11) and (28). Nevertheless, it is difficult to theoretically obtain the dynamical solutions of the discrete system. Thus, there is necessity to perform numerical simulations, the most reliable approach with satisfactory accuracy and efficiency, for comprehensively investigating the self-organized patterns of the discrete predator-prey system with feasible parameters.

In the numerical simulations, it is found that the discrete predator-prey system can exhibit two types of self-organized patterns. The first type is Turing patterns, which are selforganized under the mechanism of Turing instability, and the occurrence conditions for Turing patterns are provided in Section 3. The other type is non-Turing patterns, the emergence of which does not satisfy the Turing instability conditions. Moreover, the non-Turing patterns merely appear 
when the fixed point $\left(u_{2}, v_{2}\right)$ is unstable, i.e., the conditions in (11) are unsatisfied.

4.1. Turing Patterns. Firstly, numerical simulations are performed to demonstrate the Turing pattern formation based on the theoretical results. In the simulations, parameter values are provided based on former research works $[19,20$, 24]. Accordingly, feasible parameter ranges/values are given as follows: $Q=0.05 \sim 0.92, R=0.05 \sim 1.4, S=0.5 \sim 1.48, \tau=$ 0.01 or $0.05, l=0.25$ or $0.5, C_{1}=0.001 \sim 0.5, C_{2}=0.01 \sim$ $0.5, D_{1}=0.002 \sim 0.1, D_{2}=0.2$; unless otherwise specified, for all patterns we give $n=200, m=45000$. The initial condition for simulating pattern formation is set by perturbing $\left(u_{2}, v_{2}\right)$ using small random spatially heterogeneous perturbations. Since predator and prey patterns always show similar configuration, the results of prey patterns are merely demonstrated. Simultaneously, it should be noticed that the migration direction in all pattern graphs is set to be from up to down.

As heterogeneous perturbations take place on the spatially homogeneous states, the discrete predator-prey system may experience Turing instability and the system dynamics converges to stable spatially heterogeneous states. To demonstrate the Turing instability and pattern formation conditions, curves of $L(\bar{i})$, which is defined as $L(\bar{i})=$ $\max _{\bar{j}=1}^{n}\left\{\max \left(\left|\bar{\lambda}_{+}\right|,\left|\bar{\lambda}_{-}\right|\right)\right\}$, are plotted in Figure 1. It is shown that the curves of $L(\bar{i})$ appear like a "V" form, descending at the beginning and then rising with increase of the wavenumber $\bar{i}$. Moreover, as the values of parameters $R$ and $Q$ decrease, or the value of parameter $S$ grows, curves of $L(\bar{i})$ will go up over the critical line $L(\bar{i})=1$. With given parametric conditions in Figure 1 , when $S>0.98, R<0.6$, and $Q<0.65$, respectively, the maximum value of $L(\bar{i})$ is larger than one and Turing instability occurs, leading to the emergence of Turing patterns in the discrete predator-prey system.

Figure 2 exhibits the variation of patterns under three predator migration rates. When the migration rates of both predator and prey populations are small (Figure 2(a)), the discrete predator-prey system is close to a reaction-diffusion system and striped pattern emerges [21]. With increase of $C_{2}$ to 0.1 , the stripes in the pattern tend to be perpendicular to migration direction (Figure 2(b)). When the predator migration becomes large (Figure 2(c)), the striped pattern totally changes to be banded pattern, with less and wider bands parallel to each other. The pattern transition in Figure 2 suggests that the predator with faster migration rate may cause stronger predation effect and drive the prey forming a banded structure. The occurrence of prey bands results from the fact that prey individuals at the same parallel lines in the bands suffer similar predation pressure, and such banded structure may be beneficial to the predation of predator individuals.

With the increase of prey migration rate, Figure 3 exhibits similar dynamical phenomenon, a transition from labyrinth pattern to banded pattern. At low prey migration rate, the predator and prey patterns both present a structure of labyrinth (Figure 3(a)). The labyrinth structure is broken as the value of $C_{1}$ grows to 0.1 (Figure 3(b)), and the pattern turns to be a banded structure in which bands of prey aggregate and no individuals alternate with each other. The banded prey pattern further becomes regular at high prey migration rate due to the quick "pass-through" of more prey individuals (Figure 3(c)). However, compared with Figure 2(c), the bands in Figures 3(b) and 3(c) are parallel to the prey migration direction. Since the migration of prey may bring more than enough food, the predator can just take food in a few small patches to support the population growth. And, with the prey migrating from up to down, the predation leads to the occurrence of bands without prey individuals. Simultaneously, parallel prey bands are also self-organized by the aggregation and migration of the prey individuals which are not predated by the predator.

With the variation of parameter values, the discrete predator-prey system may show various spatiotemporal complexities. As shown in Figures 4 and 5, transitions from spot pattern to banded pattern are demonstrated with the change of predator and prey migration rates. When the migration of predator and prey is not considered, the system converges to the states of cold or hot spot patterns. As the predator or prey migration rate rises, the clod/hot spots in the patterns gradually connect with each other to form fine stripes, resulting in the emergence of a type of intermediate patterns, spot-stripe patterns. As migration effect of predator or prey continuously enhances, the spot-stripe patterns transit to patterns of stripes; then the width of prey stripes increases, and the striped patterns turn to be replaced by the banded patterns.

4.2. Non-Turing Patterns. Figure 6 also exhibits the transition of patterns from hot spots to spots-stripes and then to banded pattern with the increase of predator migration rate. However, the pattern formation in this case is very different from that in Figures 2-5. As shown in Figure 7(a), when we do not consider the diffusion and migration of both predator and prey populations, the nonspatial predator-prey system will converge to $\left(u_{1}, v_{1}\right)$, which is a state without coexistence of predator and prey, but with predator extinct and prey reaching carrying capacity. The results in Figures 6 and 7 suggest that the diffusion and migration of populations can lead to pattern self-organization and improve population coexistence in the discrete predator-prey system. The conditions for pattern self-organization of this case are different from the two conditions described in Section 3 for the Turing patterns. Therefore, we here find the emergence of a type of non-Turing patterns, which help to better comprehend more possible ways for the coexistence of predator and prey in space. Moreover, Figures 7(b)-7(d) show that the dynamics of predator and prey is always periodically oscillating with time. And as the predator migration rate enhances, the frequency of the periodic oscillation gradually goes up. This suggests that the patterns in Figure 6 are migrating with time, and the pattern migrating speed is positively related to the population migration rate.

The emergence of non-Turing patterns can also take place in other cases, as shown in Figures 8 and 9. With the parametric conditions given in Figure 8, when we study the nonspatial dynamics of the discrete predator-prey system, 


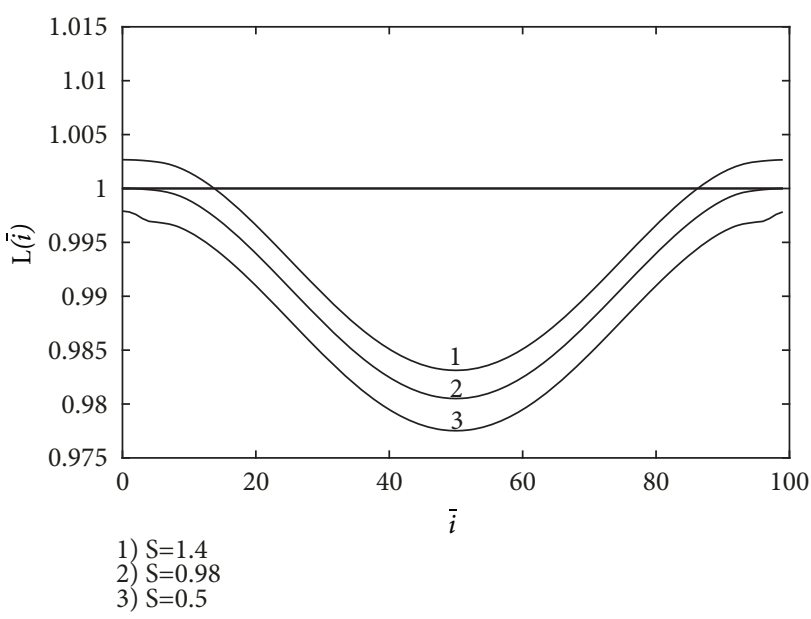

(a)

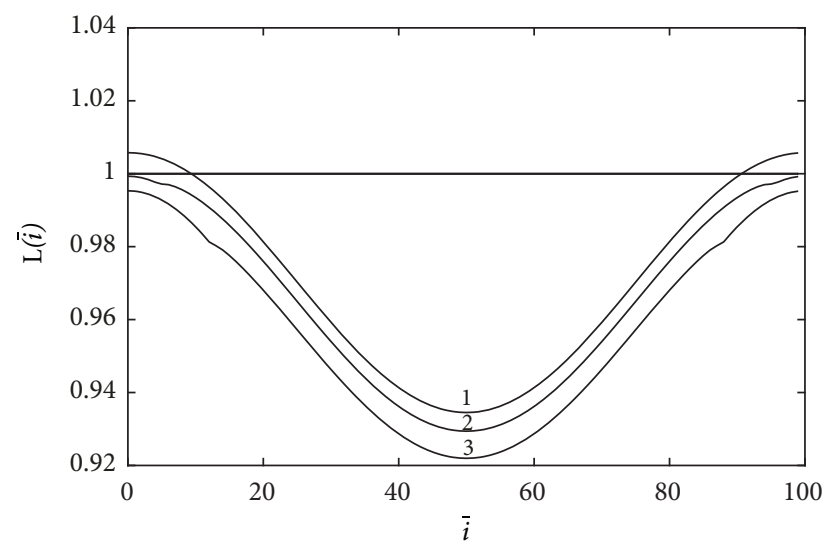

1) $\mathrm{R}=0.05$

2) $\mathrm{R}=0.6$

(b)

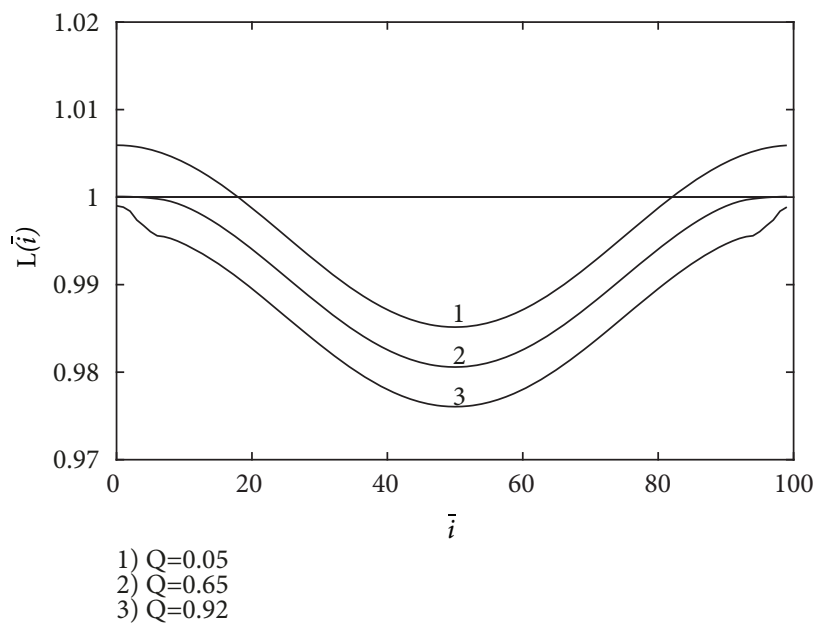

(c)

FIGURE 1: Display of Turing instability with variation of system parameters. Parametric conditions are given as $\tau=0.01, l=0.25, C_{1}=0.1, C_{2}$ $=0.1, D_{2}=0.2$, (a) $Q=0.6, D_{1}=0.02, R=0.5$, (b) $Q=0.6, S=1.1, D_{1}=0.1$, (c) $S=1.1, D_{1}=0.02, R=0.5$.

i.e., do not consider the population movement, both predator and prey will be extinct eventually, suggesting the collapse of the predator-prey system (Figures 9(a) and 9(b)). However, with diffusion and migration, the predator and prey can coexist (Figures 9(c)-9(k)) and are self-organized in spiral or banded patterns in space (Figure 8). The reason for predatorprey coexistence and pattern self-organization in such case is that the population migration continuously brings new individuals, which supports the maintenance of the predatorprey system.

The occurrence of two types of patterns in Figure 8, spiral and banded patterns, is determined by the contrast of the predator and prey migration. First, comparatively equal migration rates of predator and prey lead to the formation of spiral patterns. In this situation, the predator and prey individuals migrating into every site per unit time always keep synchronous. This says that the migration effects of predator and prey just counteract and the population diffusion dominates in the pattern self-organization process.
Second, banded pattern occurs when the migration rates of predator and prey have difference, i.e., the predator and prey migrations are out of sync. Moreover, with the increase of population migration rates $\left(C_{1} \neq C_{2}\right)$, the number of bands in the prey pattern grows. On the other hand, the pattern migrating speed is influenced by the difference between $C_{1}$ and $C_{2}$, as shown in Figures 9(c)-9(k). Generally, the lowest migrating rate of prey patterns takes place at the situations of $C_{1}=C_{2}$.

Figure 10 shows more cases of non-Turing patterns. Similarly to Figure 8, the nonspatial system also converges to the state with the absence of predator and prey. Under the effect of migration, the predator-prey system continues with the coexistence of both predator and prey populations. The pattern self-organization in Figure 10 exhibits three aspects of characteristics. First, when the migration rates of predator and prey are equal, the prey will be self-organized into a pattern with irregular and disordered patches. As shown in the pattern configurations of Figures 10(a), 10(f), 10(k), 10(p), 


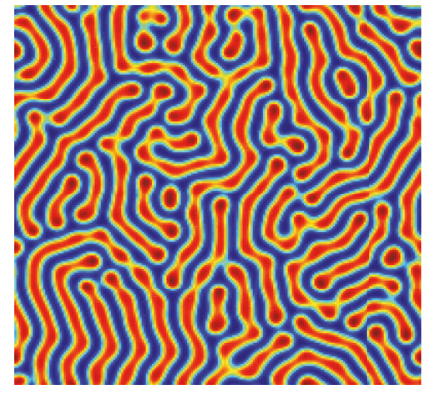

(a) $C_{2}=0.01$
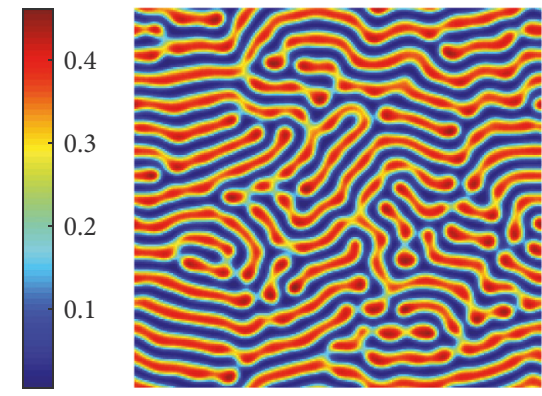

(b) $C_{2}=0.1$

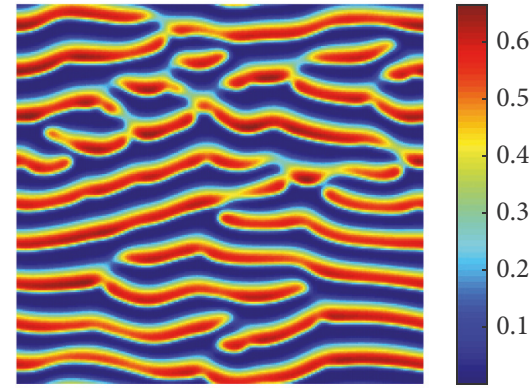

(c) $C_{2}=0.3$

FIGURE 2: Variation of prey patterns under three predator migration rates. With the increase of $C_{2}$, a change is shown from striped pattern to banded pattern. $Q=0.6, R=0.5, S=1.1, \tau=0.01, l=0.25, C_{1}=0.005, D_{1}=0.015, D_{2}=0.2$.

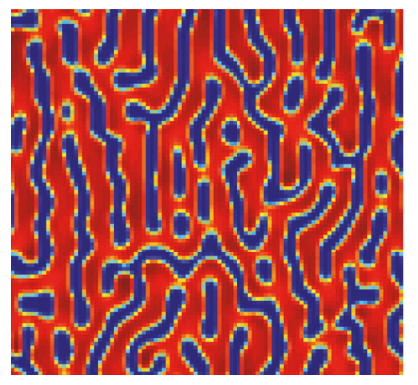

(a) $C_{1}=0.01$
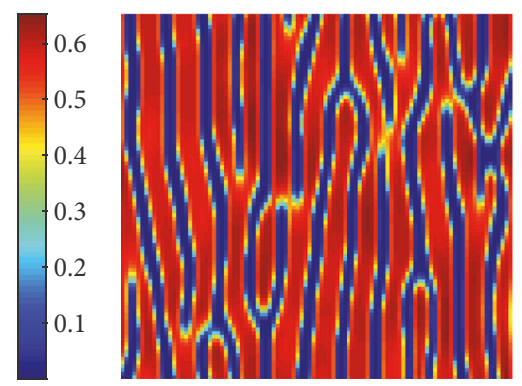

(b) $C_{1}=0.1$
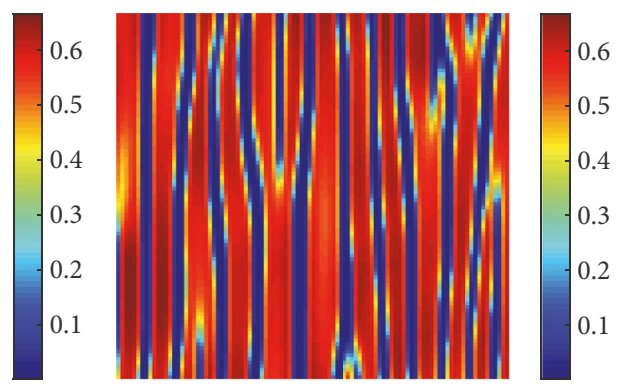

(c) $C_{1}=0.5$

FIGURE 3: Variation of prey patterns under three prey migration rates. With the increase of $C_{1}$, a change is shown from labyrinth pattern to banded pattern. $Q=0.6, R=0.5, S=1.1, \tau=0.01, l=0.25, C_{2}=0.01, D_{1}=0.002, D_{2}=0.5, n=100$.

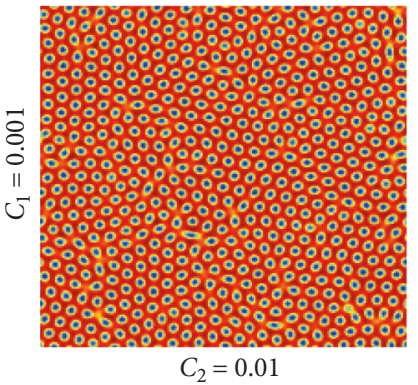

(a)

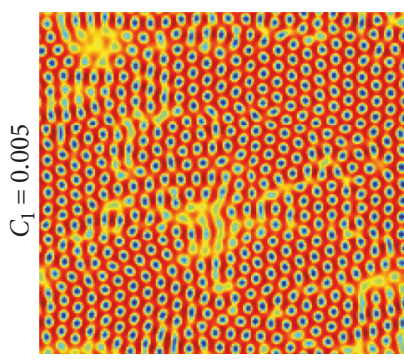

$C_{2}=0.01$

(d)
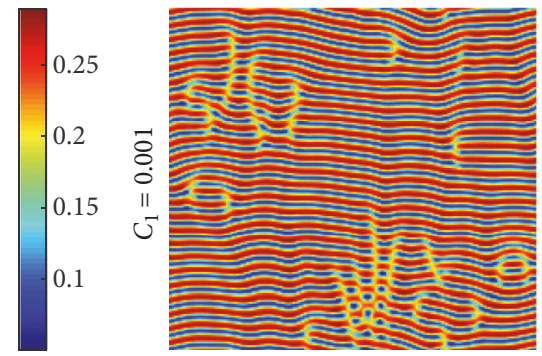

$C_{2}=0.1$

(b)

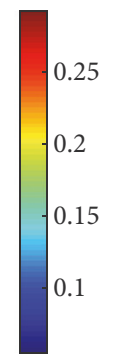

$$
\text { 各 }
$$

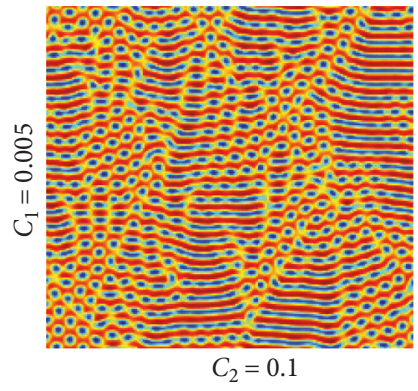

(e)
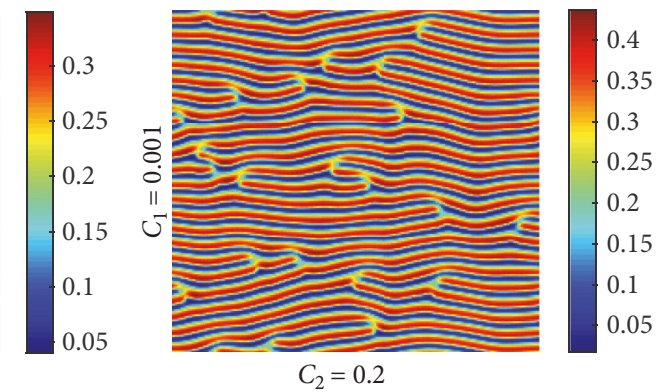

(c)
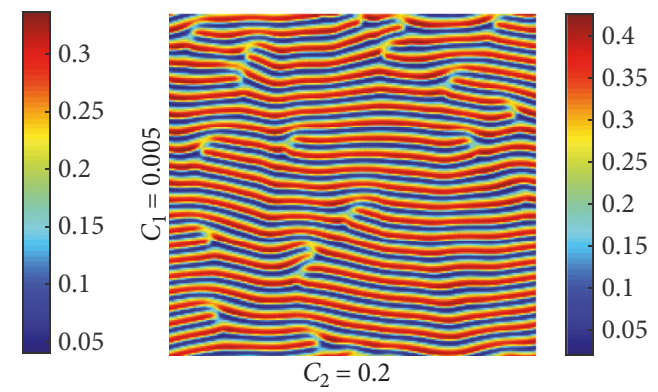

(f)

FIGURE 4: Variation of prey patterns with the change of predator and prey migration rates. Transitions from cold spot pattern to banded pattern are demonstrated. $Q=0.6, R=0.5, S=0.98, \tau=0.05, l=0.5, D_{1}=0.02, D_{2}=0.2$. 


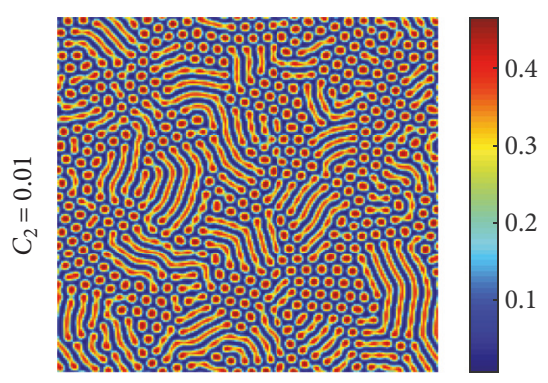

$C_{1}=0.001$

(a)

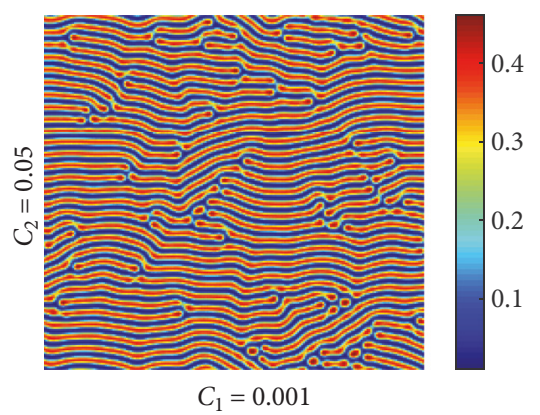

(d)

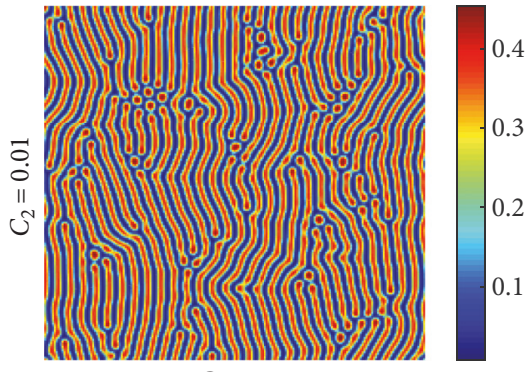

$C_{1}=0.005$

(b)

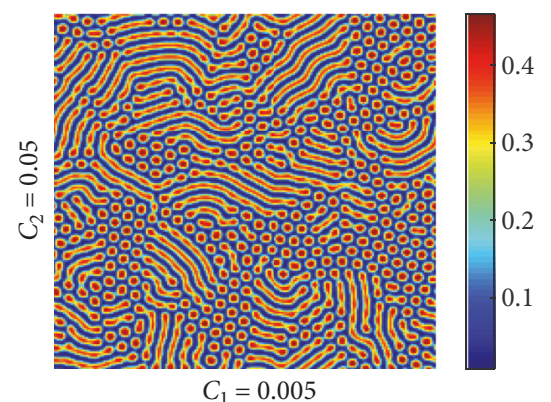

(e)

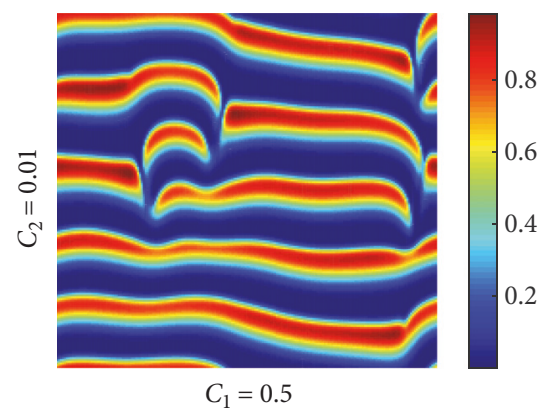

(c)

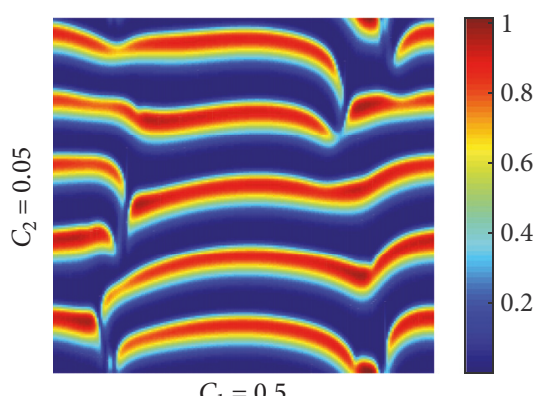

(f)

FIGURE 5: Variation of prey patterns with the change of predator and prey migration rates. Transitions from hot spot pattern to banded pattern are demonstrated. $Q=0.6, R=0.5, S=1.15, \tau=0.05, l=0.5, D_{1}=0.02, D_{2}=0.2$.

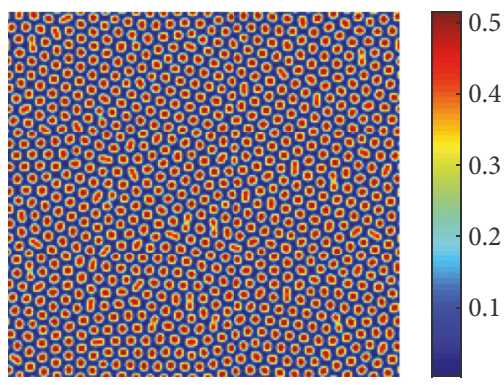

(a) $C_{2}=0.01$

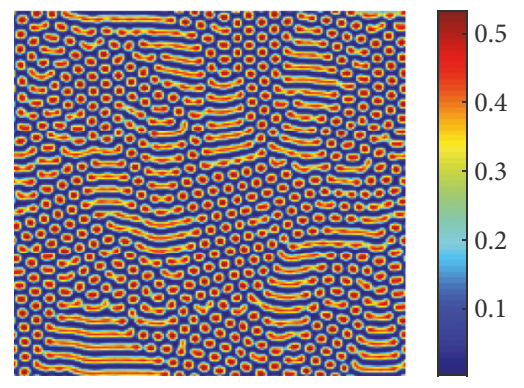

(b) $C_{2}=0.1$

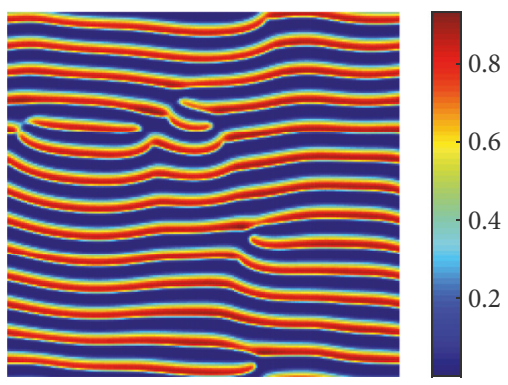

(c) $C_{2}=0.5$

Figure 6: Transition of prey patterns from hot spots and spots-stripes to banded pattern with the increase of predator migration rate. The patterns self-organized in this case are non-Turing patterns. $Q=0.6, R=0.5, S=1.24, \tau=0.05, l=0.5, C_{1}=0.005, D_{1}=0.02, D_{2}=0.2$.

a few irregular prey patches just emerge disorderedly on blue background, which suggests no prey individuals. Second, different migration rates of predator and prey result in the self-organization of banded prey patterns. On the one hand, when $C_{1}$ and $C_{2}$ show small difference, such as 0.05 and 0.1 or 0.01 and 0.05 , the system may produce irregular banded patterns, in which the prey bands may show fragmented and curved properties. On the other hand, regular banded patterns are generated if the difference between the values of $C_{1}$ and $C_{2}$ is large, such as 0.5 and 0.1 . Third, the change of band number and pattern migrating speed with the variations of $C_{1}$ and $C_{2}$ values is similar to that in Figure 8.

The irregularity may not only be shown in the pattern configuration, but also be reflected in the pattern selforganization process. Figure 11 is plotted to check the irregularity in the pattern self-organization via sensitivity analysis, with the application of two close initial conditions. The difference quantity between these two initial conditions is merely at one site in the prey pattern, given as 0.0001 . Then we randomly select another site in the prey pattern and study the difference of the dynamics induced by the two close initial conditions, i.e., $P_{u}(t)=u_{1\left(i_{1}, j_{1}, t\right)}-u_{2\left(i_{1}, j_{1}, t\right)}$, in which $\left(i_{1}, j_{1}\right)$ represents a random site. As time progresses, we find that for the irregular patterns, tiny difference in initial conditions can lead to large divergence of pattern self-organization processes (see Figures 11(a)-11(b), 11(e)-11(g), 11(j)-11(k) and $11(\mathrm{p})$ ), indicating the formation of two different patterns. Such dynamic divergence may suggest spatiotemporal chaos which takes place in the process of pattern self-organization. We also perform sensitivity analysis on the patterns in Figures $2,3,4,5,6$, and 8 and the regular patterns in Figure 10, and we find that the tiny difference in initial conditions will not 

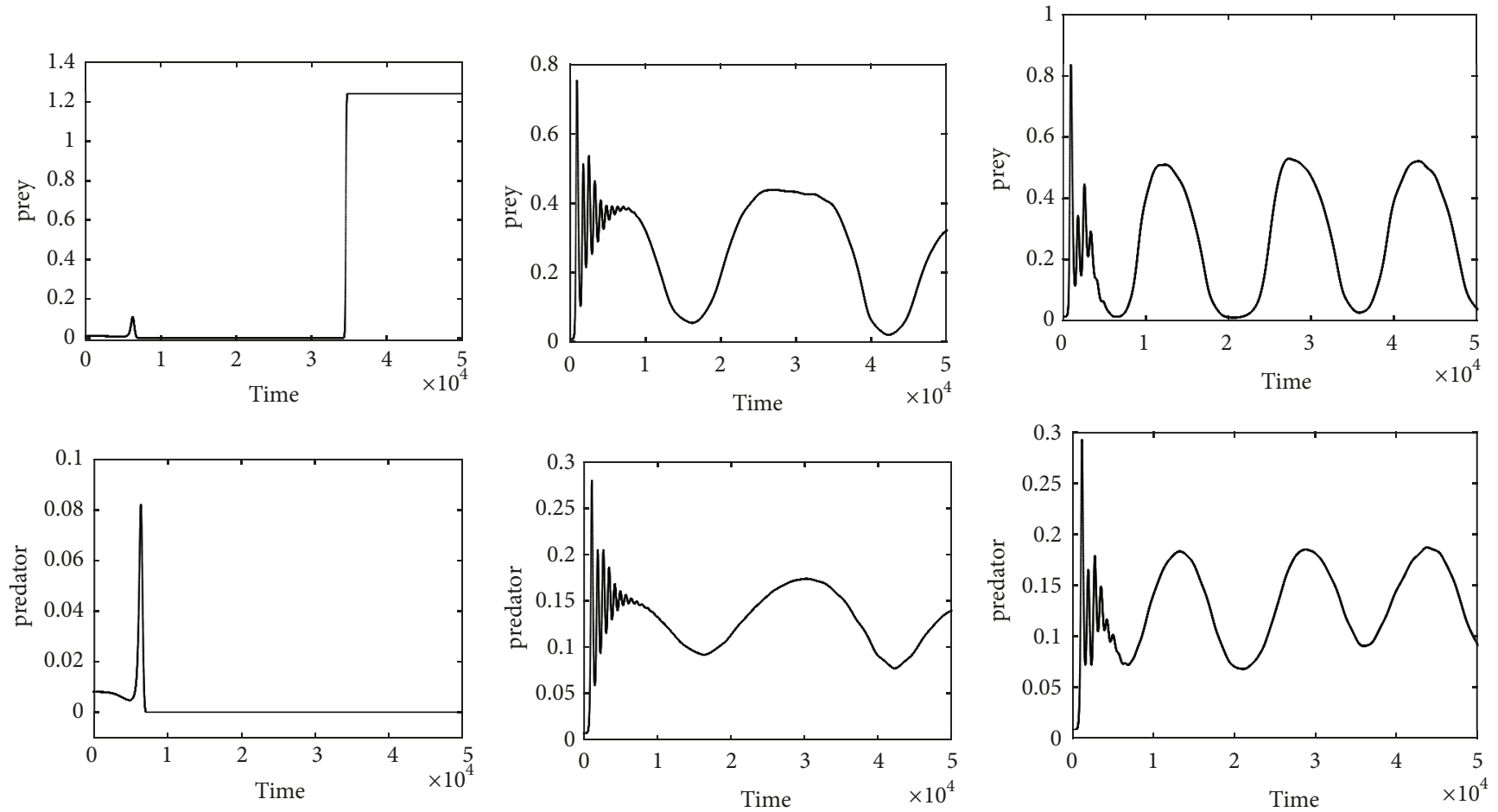

(a)

(b)

(c)
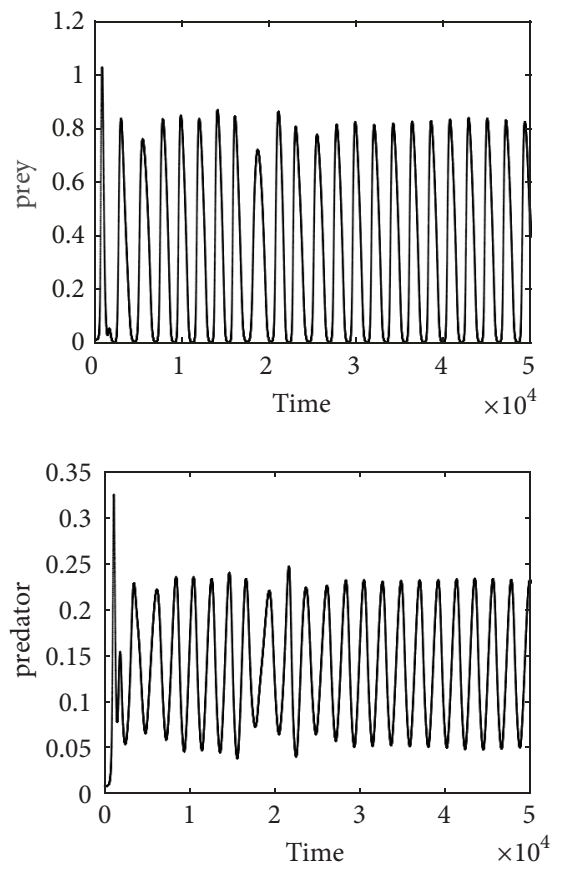

(d)

Figure 7: Temporal dynamics of predator and prey corresponding to (a) the nonspatial case and (b)-(d) the three pattern self-organization cases in Figure 6, respectively. The graphs (b)-(d) are plotted using the dynamics of grid cell $(100,100)$ in the pattern (the same for Figure 9). Parametric conditions are the same with that in Figure 6. 


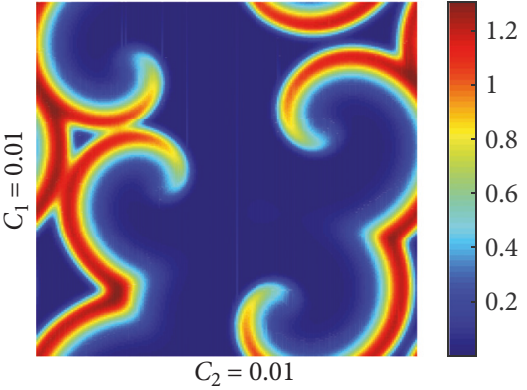

(a)

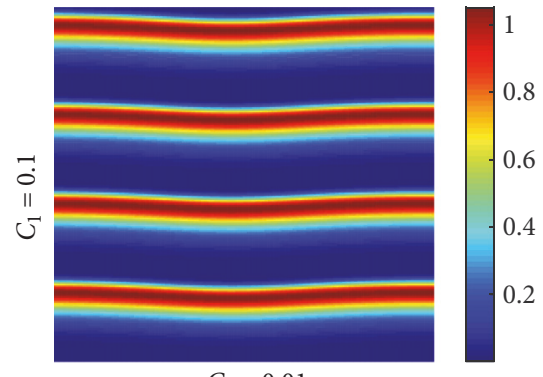

(d)

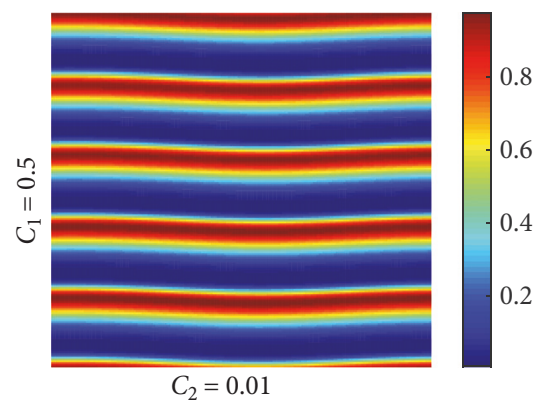

(g)

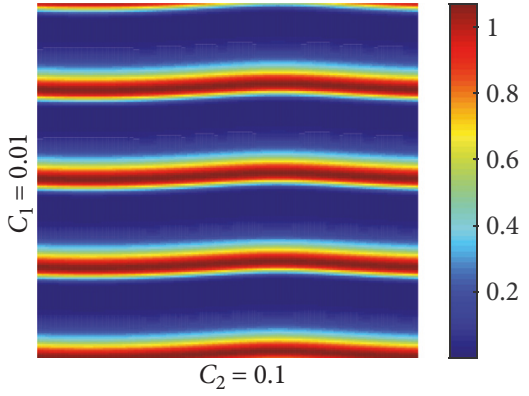

(b)

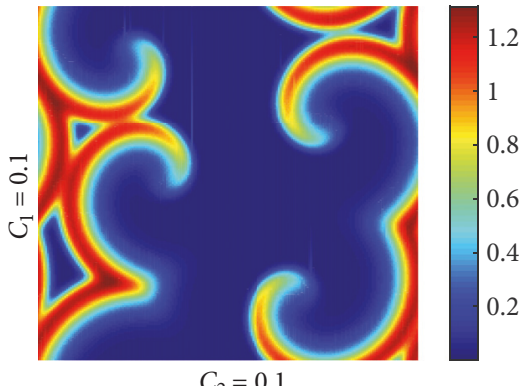

(e)

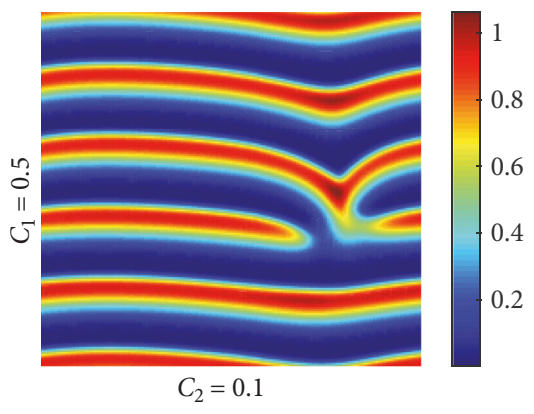

(h)

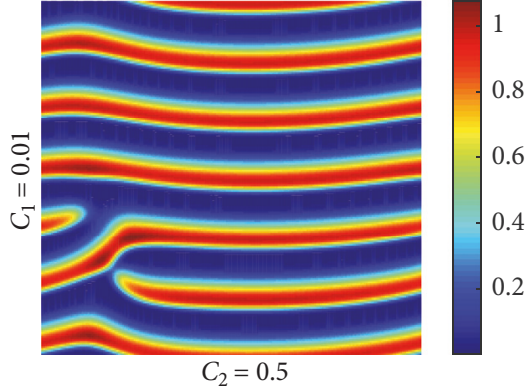

(c)

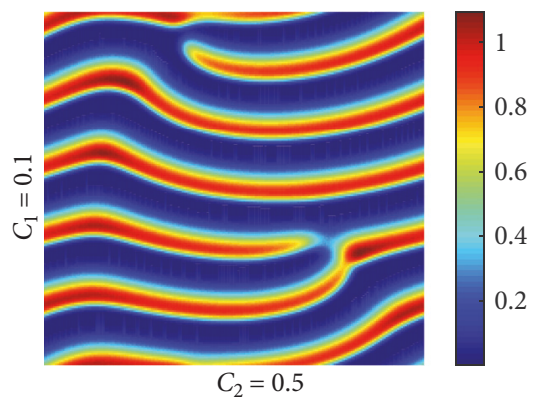

(f)

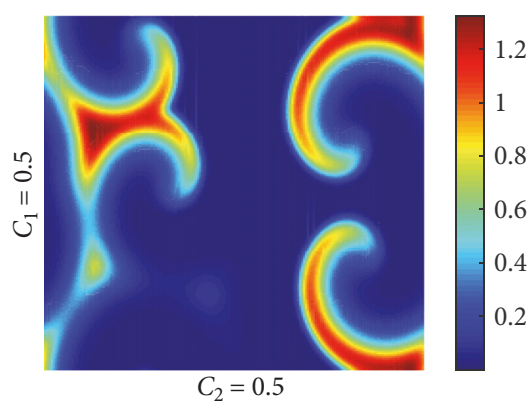

(i)

FIGURE 8: Variation of prey patterns with the change of predator and prey migration rates. Two types of non-Turing patterns, spiral and banded patterns, are self-organized in this case. $Q=0.55, R=0.75, S=1.48, \tau=0.05, l=0.5, D_{1}=0.2, D_{2}=0.2$.

be amplified or restrained in a very small level as time grows. This suggests that these patterns are difficult to be changed by small external disturbances.

\section{Discussion and Conclusions}

Exploring the spatiotemporal complexity of predator-prey systems with the application of CMLs is one of the important topics in the fields of mathematical biology and ecology [2327]. As described in literature, the most important characteristic of the CMLs is the processes segregation, which may be the key for better exhibiting spatiotemporal complexity [26]. To explore the pattern self-organization in a predatorprey system under the effects of migration and diffusion, a new three-chain CML model is developed in this research via segregating the ecological processes of reaction, diffusion, and migration based on ecological reality. It should be noticed that applying three-chain CML for studying the predatorprey systems has never been documented in literature.
Via Turing instability analysis and numerical simulations on the three-chain CML, rich and complex Turing patterns are revealed for the spatiotemporally discrete predator-prey system studied in this research. Generally, the Turing patterns are self-organized under two mechanisms, i.e., diffusiondriven and migration-driven mechanisms. When the migration of both predator and prey is weak, the diffusion plays the most important role in pattern self-organization. In such case, the discrete predator-prey system exhibits rich patterns with subtle self-organized structures, such as stripes, labyrinth, hot spots, cold spots, and stripes-spots. Nevertheless, when the population migration becomes dominant, the system converges to merely one type of states, banded patterns.

Moreover, a new result, the self-organization of nonTuring patterns, is found for the spatiotemporal complexity of the predator-prey system with ratio-dependent functional response. It suggests that even in the cases where the nonspatial predator-prey system reaches collapse, the migration can still maintain the coexistence of predator and prey and lead 


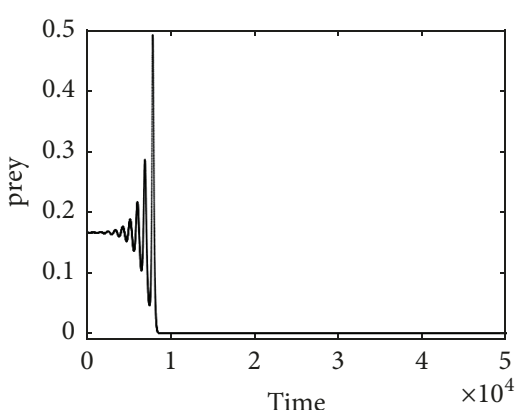

(a)

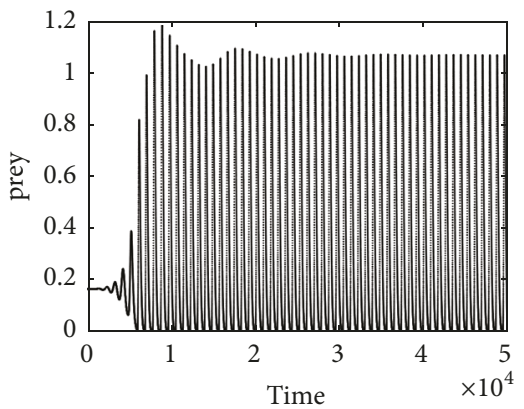

(d)

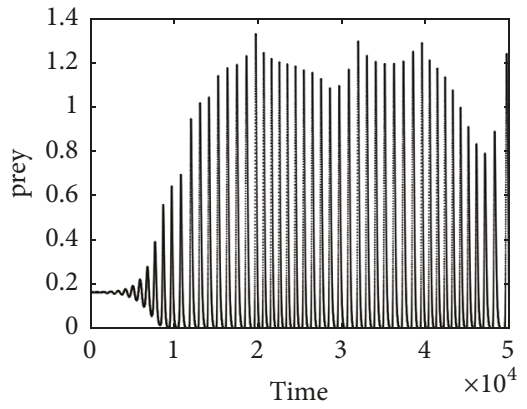

(g)

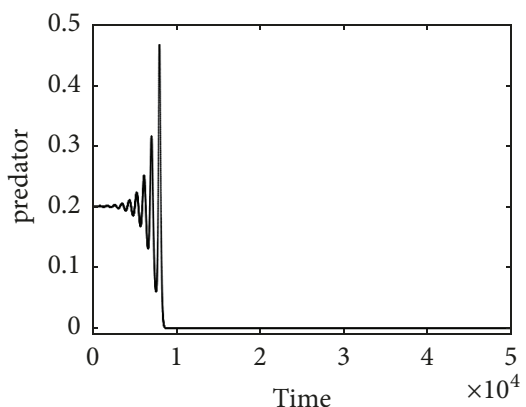

(b)

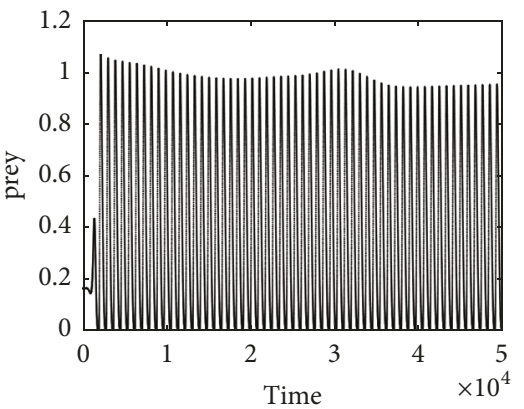

(e)

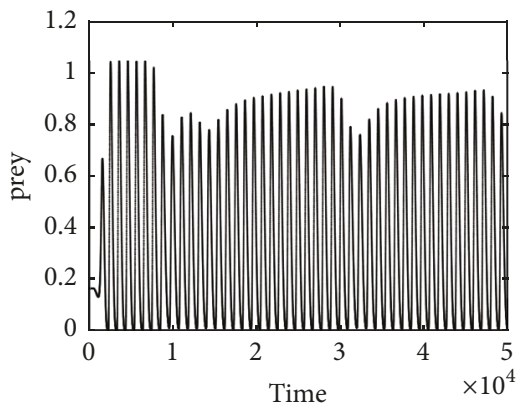

(h)

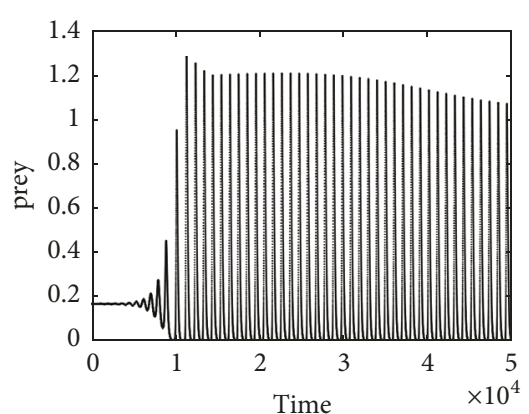

(c)

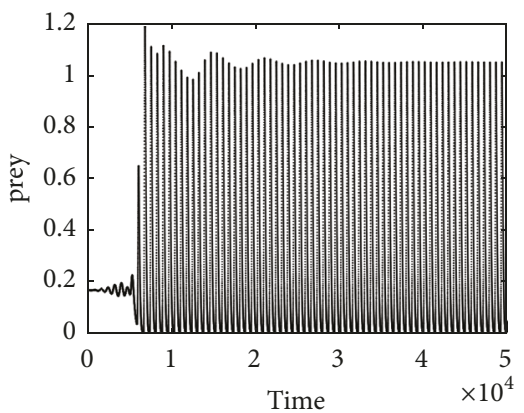

(f)

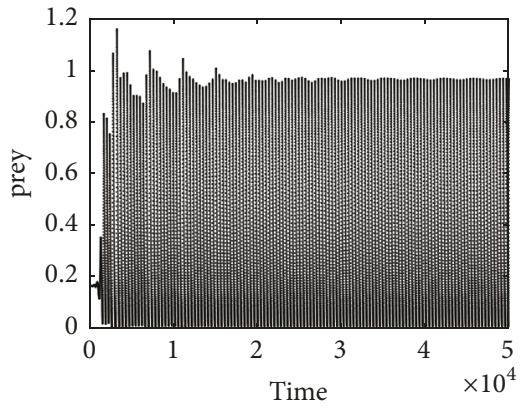

(i)

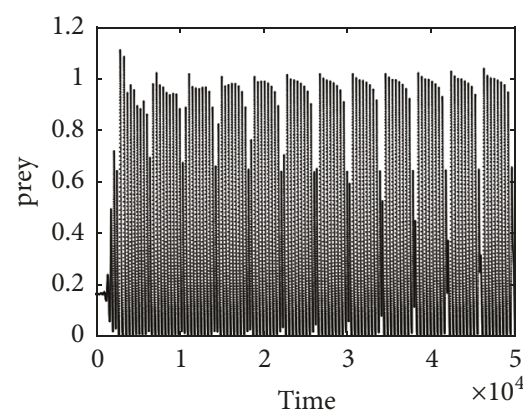

(j)

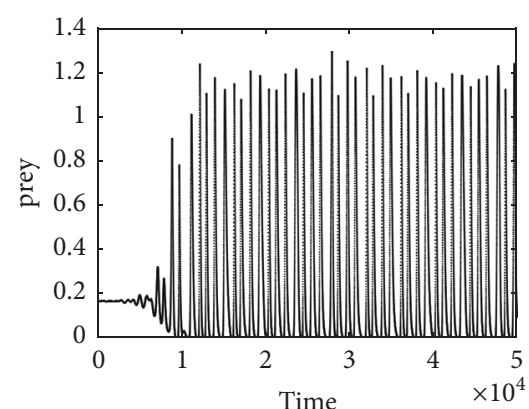

(k)

FiguRE 9: Temporal dynamics of predator and prey corresponding to (a)-(b) the nonspatial case and (c)-(k) the pattern self-organization cases in Figure 8, respectively. Parametric conditions are the same as that in Figure 8.

to pattern self-organization. Here, the migration contributes to continuously imputing new individuals which cause the populations in the predator-prey system not to be extinct. The non-Turing patterns may emerge in two cases, where the nonspatial system shows extinction of (1) the predator and (2) both the predator and the prey. In the first case (Figure 6), dynamic transition from spotted pattern to banded pattern can also be observed. For the second case (Figures 8 and 10), the non-Turing patterns demonstrate three types in configuration as the migration rates fluctuate, i.e., banded, spiral, and irregular patterns. From the simulation results, it is found that the difference between the predator and prey migration rates controls the occurrence of pattern types. When the predator and prey migration rates show explicit difference, 


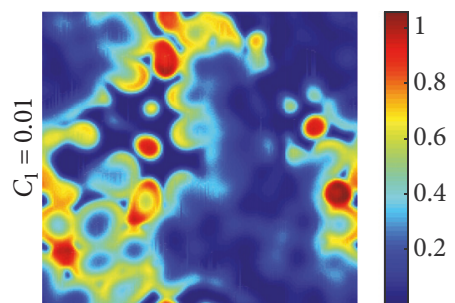

$C_{2}=0.01$

(a)

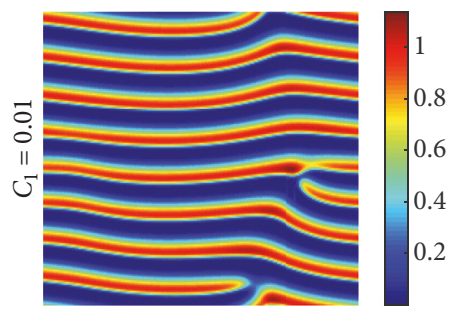

$C_{2}=0.5$

(d)

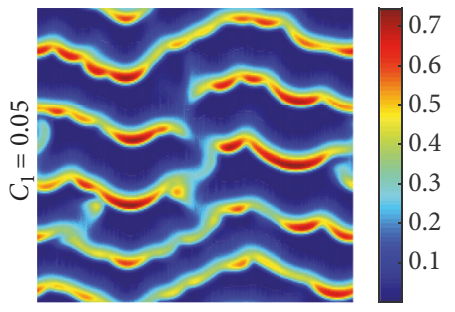

$C_{2}=0.1$

(g)

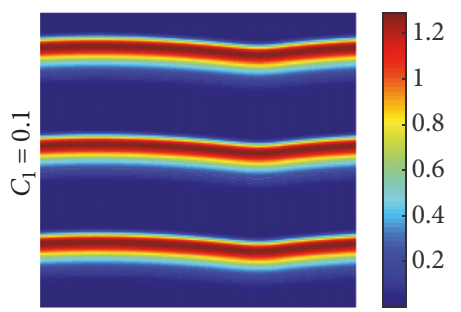

$C_{2}=0.05$

(j)

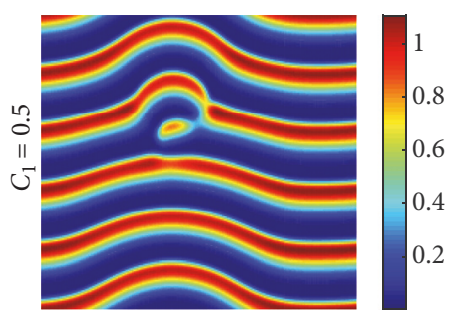

$C_{2}=0.01$

(m)

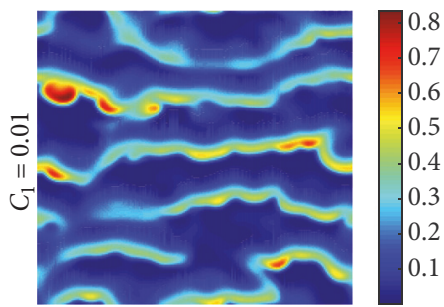

$C_{2}=0.05$

(b)

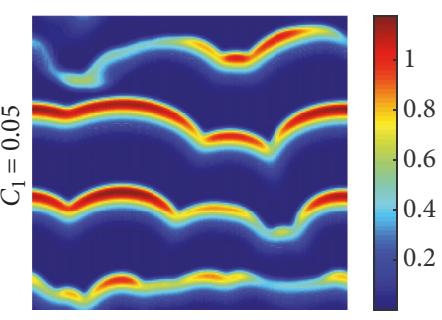

$C_{2}=0.01$

(e)

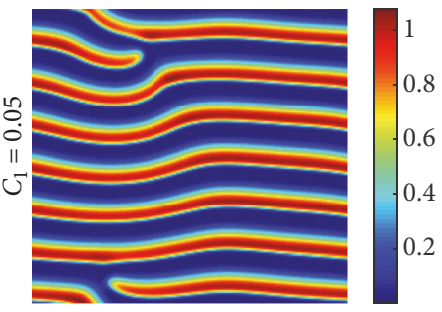

$C_{2}=0.5$

(h)

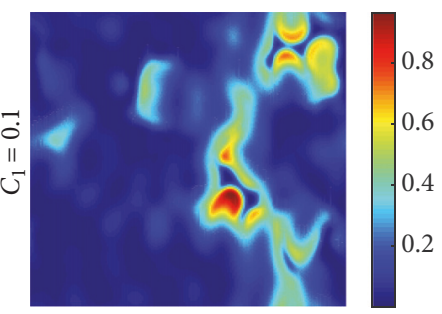

$C_{2}=0.1$

(k)

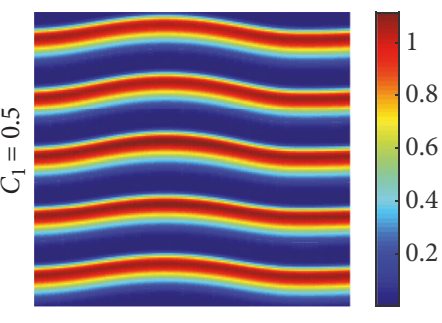

$C_{2}=0.05$

(n)

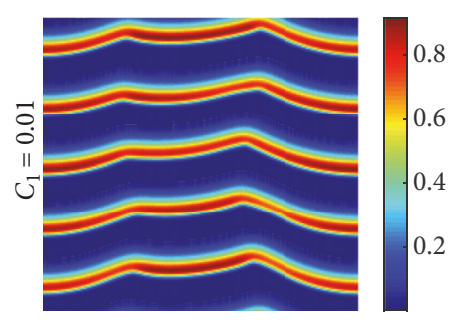

$C_{2}=0.1$

(c)

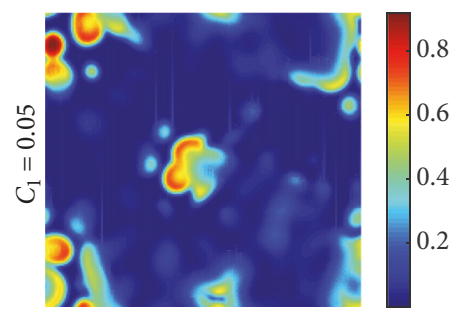

$C_{2}=0.05$

(f)

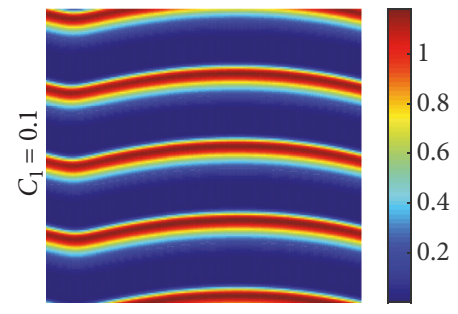

$C_{2}=0.01$

(i)

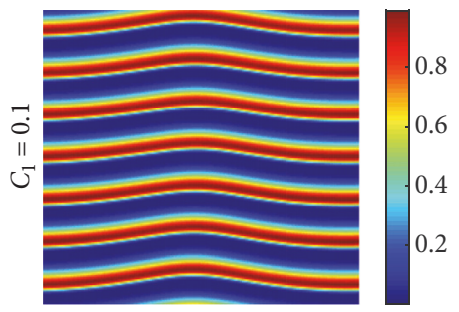

$C_{2}=0.5$

(1)

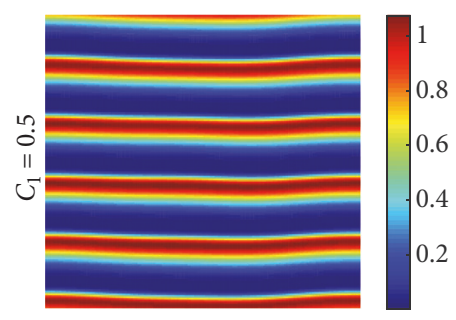

$C_{2}=0.1$

(o)

FIgure 10: Continued. 


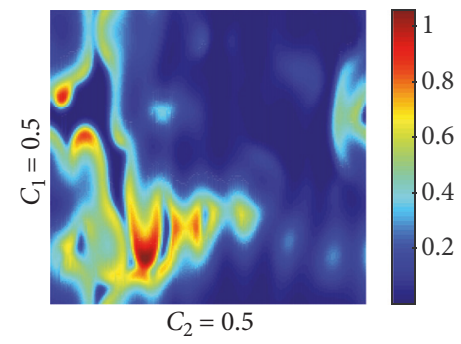

(p)

FIGURE 10: Variation of prey patterns with the change of predator and prey migration rates. These cases are also non-Turing patterns, in which the prey is self-organized into irregular disordered patches, irregular and regular bands. $Q=0.55, R=0.76, S=1.48, \tau=0.05, l=0.5, D_{1}=0.1$, $D_{2}=0.2$.

banded patterns prevail. Moreover, with the increase of either predator or prey migration rates, the number of bands in the pattern rises up. When the migration rates of predator and prey become close, the discrete predator-prey system mainly exhibits spiral or irregular patterns. The irregular patterns have the characteristics of spatiotemporal chaos, suggesting different initial conditions can result in the self-organization of diverse patterns.

The various patterns revealed by the CML suggest that the predator and prey populations can exhibit diverse spatial distribution when the populations reach different habitats which restrict or promote the migration and diffusion of the predator and the prey. It should be noticed that the spotted, striped, spiral, and banded patches in the prey patterns, which are selforganized after long-term predator-prey interactions, play a role of refuge for the prey individuals, since the predator densities in these patches often hold at a low level. Contrarily, the regular or irregular patches in the predator patterns are the gathering areas of the predator individuals. This may explain the situations where the populations often live in stable settlements in nature. As the populations migrate, the patterns can keep unchanged and, most importantly, move forward in the direction of migration. This suggests that gathering behaviors of the predator and the prey evolve from long-term predator-prey interactions and may not be altered during the migration process. The pattern diversity predicts a lot of possible stably coexistent ways for the predator and prey in different habitats. Moreover, the transitions of patterns may reflect stability and resilience of the predator-prey system to environmental changes along the migration route. From the transition between diffusion-dominant and migrationdominant patterns, one can predict whether the local habitats show attraction to prey and predator populations.

Banded patterns are a type of special phenomenon widespreadly existing in nature. In the present study, the banded patterns exhibited by the CML model show two types. First, when predator migration prevails, the prey bands in the predator and prey patterns are perpendicular to the migration direction. In this case, the occurrence of such prey bands may result from the predator driving the prey migrating synchronously. Second, when the prey migration prevails, the patterns of predator and prey show bands parallel or perpendicular to the migration direction. The emergence of parallel banded patterns may be caused by the migration of prey providing sufficient food for the predator. Both types of banded patterns reveal the best redistribution of predator and prey individuals for predation and population growth under the effects of migration. In nature, the banded pattern selforganization in predator-prey systems has been found. For example, researchers have observed regular banded patterns of mussel beds in the Wadden Sea of the Netherlands [40]; in this system, algae population moves by advection (i.e., the prey migration rate is large) and mussel population, living in the same environment, mainly moves by diffusion (i.e., the predator migration rate is zero or tiny). Moreover, the bands in the mussel patterns are perpendicular to the prey migration direction, coincident with the property of the second type of banded patterns as described above.

Spiral and irregular spatiotemporal patterns, which are predicted as non-Turing patterns in the present study, have important implications for population dynamics and are widely regarded to be of high ecological relevance [41]. For example, it is well known that the distribution of plankton can be highly heterogeneous under the interactions of phytoplankton-zooplankton-fish [42], and the movement of phytoplankton and zooplankton populations with different velocities can give rise to spatial patterns [43]. During the period of plankton blooms, the spatial patterns of planktons may demonstrate an important characteristic, spatiotemporal chaos, which leads to highly unpredictable plankton dynamics [44]. However, it is also argued that ecological systems with chaotic dynamics have a greater potential for adapting to changing environmental conditions than nonchaotic ones [44].

In comparison with previous works, such as the research of Sun et al. [2] and Liu [20], the three-chain CML reveals new nonlinear characteristics and enriches the spatiotemporal complexity of predator-prey systems. Firstly, a new case, nonTuring patterns, is found, demonstrating that diffusion and migration can promote the maintenance of the predatorprey system, revealing many new more possible ways for the coexistence of predator and prey. Secondly, as studied by Sun et al. [2] and Liu [20], they found that the continuous model always exhibits traveling banded patterns once the population migration participates in pattern self-organization. Contrarily, the present study finds that the effect of diffusion 


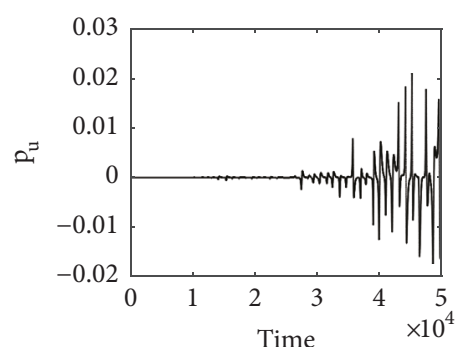

(a)

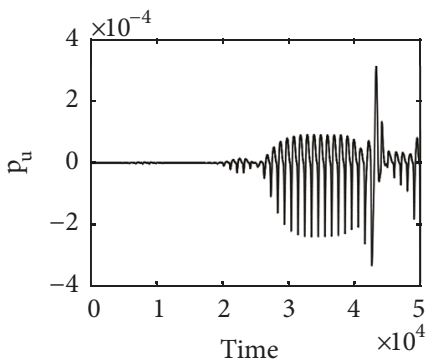

(d)

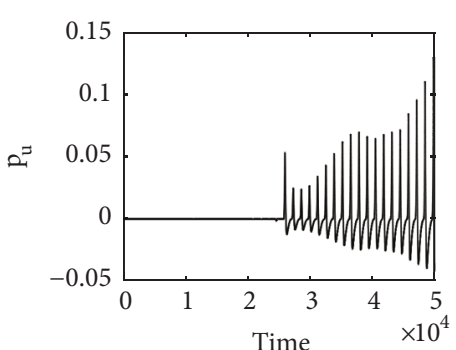

(g)

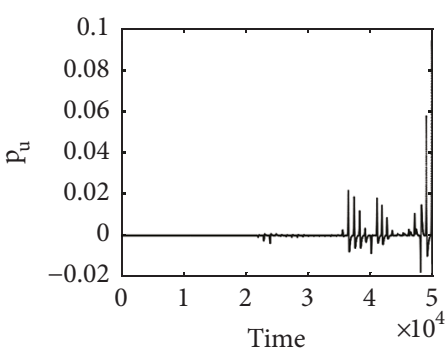

(j)

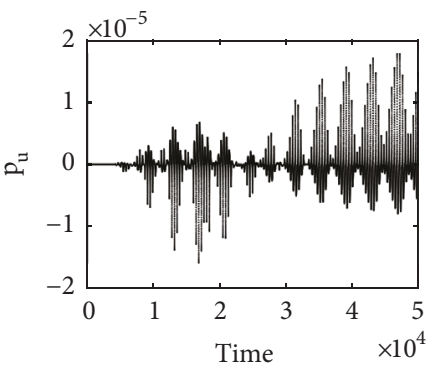

(m)

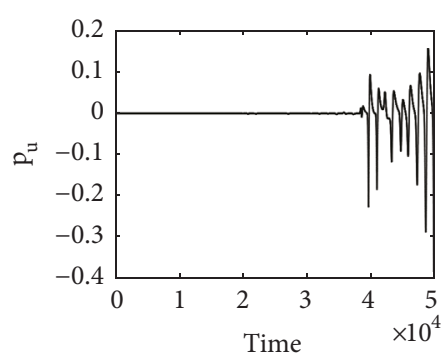

(b)

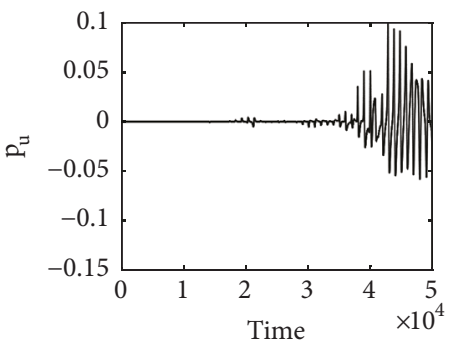

(e)

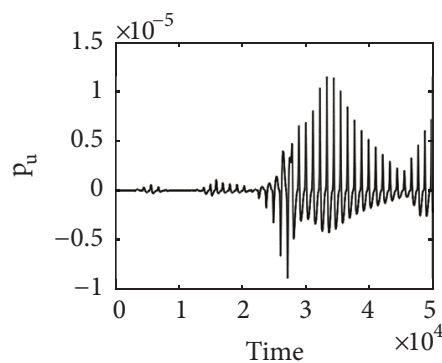

(h)

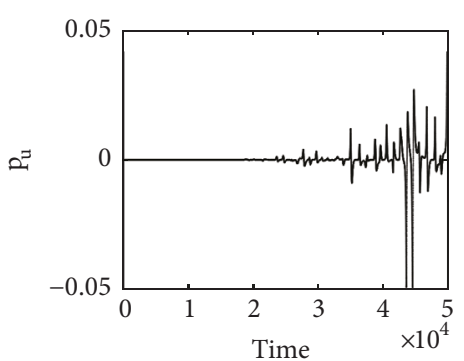

(k)

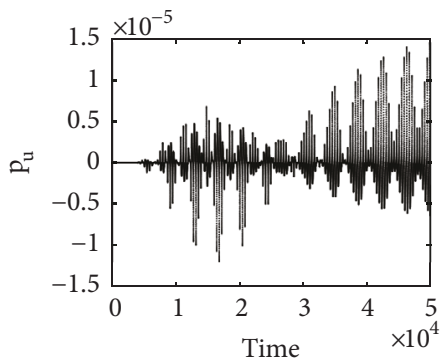

(n)

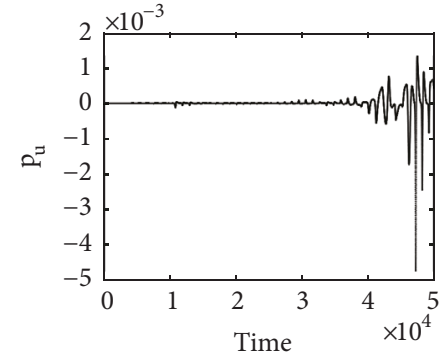

(c)

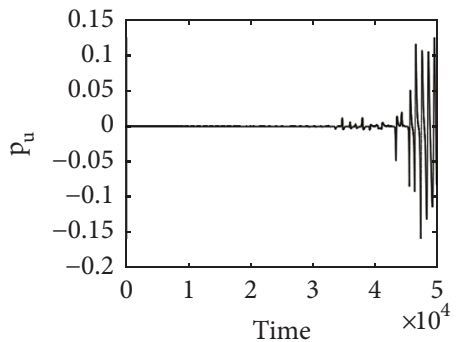

(f)

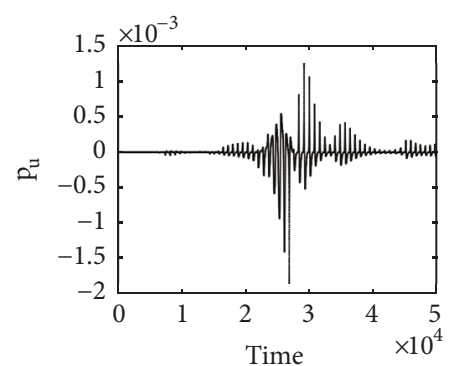

(i)

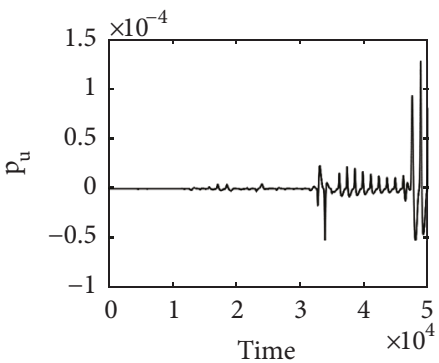

(1)

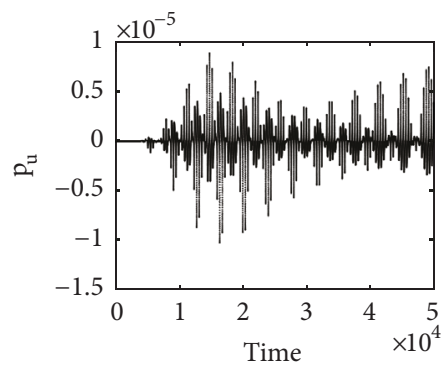

(o)

Figure 11: Continued. 


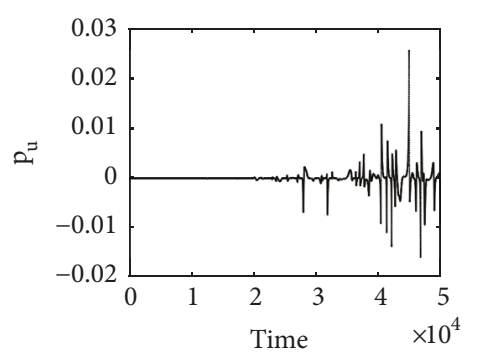

(p)

FIGURE 11: Sensitive analysis corresponding to the pattern self-organization cases in Figure 10, respectively. Parametric conditions are the same as that in Figure 10.

cannot be wholly covered and a gradual transition can appear between diffusion-driven and migration-driven patterns with the change of population migration or diffusion rate.

A few former research works have also explored the spatiotemporal complexity of the discrete predator-prey systems with the application of CML [22, 24-26, 32]. Nevertheless, with comparison, the present study shows two aspects of improvement. First, under the combination effect of diffusion and migration, the diffusion-driven and migration-driven mechanisms are specifically explored for the self-organization of Turing and non-Turing patterns. More importantly, we develop the two-chain CML into a three-chain model for investigating pattern self-organization in the discrete predator-prey system with diffusion and migration. Similarly in future works, $n$-chain CML can be generalized to explore the ecological systems with more complex segregating ecological processes.

\section{Data Availability}

The data used to support the findings of this study are included within the article.

\section{Conflicts of Interest}

The authors declare no conflicts of interest.

\section{Acknowledgments}

This research was financed by the National Water Pollution Control and Treatment Science and Technology Major Project (No. 2015ZX07204-007, No. 2017ZX07101-002), the National Natural Science Foundation of China (No. 11802093), and the Fundamental Research Funds for the Central Universities (No. JB2017069).

\section{References}

[1] M. Banerjee, "Self-replication of spatial patterns in a ratiodependent predator-prey model," Mathematical and Computer Modelling, vol. 51, no. 1, pp. 44-52, 2009.

[2] G.-Q. Sun, Z. Jin, Q.-X. Liu, and L. Li, "Dynamical complexity of a spatial predator-prey model with migration," Ecological Modelling, vol. 219, no. 1-2, pp. 248-255, 2008.
[3] R. S. Cantrell and C. Cosner, Spatial Ecology via ReactionDiffusion Equations, John Wiley \& Sons, England, UK, 2003.

[4] A. J. Lotka, The Elements of Physical Biology, Williams \& Wilkins, Baltimore, Md, USA, 1925.

[5] V. Volterra, "Fluctuations in the abundance of a species considered mathematically," Nature, vol. 118, no. 2972, pp. 558-560, 1926.

[6] M. R. Garvie, "Finite-difference schemes for reaction-diffusion equations modeling predator-prey interactions in MATLAB," Bulletin of Mathematical Biology, vol. 69, no. 3, pp. 931-956, 2007.

[7] E. Hernandezmartinez, H. Puebla, T. Perezmunoz et al., "Spatiotemporal dynamics of telegraph reaction-diffusion predatorprey models," in International Symposium on Mathematical and Computational Biology, pp. 268-281, 2012.

[8] A. M. Turing, "The chemical basis of morphogenesis," Philosophical Transactions of the Royal Society of London. Series B, Biological Sciences (1934-1990), vol. 237, no. 641, pp. 37-72, 1952.

[9] L. A. Segel and J. L. Jackson, "Dissipative structure: an explanation and an ecological example," Journal of Theoretical Biology, vol. 37, no. 3, pp. 545-559, 1972.

[10] S. A. Levin and L. A. Segel, "Hypothesis for origin of planktonic patchiness," Nature, vol. 259, no. 5545, p. 659, 1976.

[11] W.-M. Ni and M. Tang, "Turing patterns in the Lengyel-Epstein system for the CIMA reaction," Transactions of the American Mathematical Society, vol. 357, no. 10, pp. 3953-3969, 2005.

[12] F. Q. Yi, J. J. Wei, and J. P. Shi, "Bifurcation and spatiotemporal patterns in a homogeneous diffusive predator-prey system," Journal of Differential Equations, vol. 246, no. 5, pp. 1944-1977, 2009.

[13] H.-B. Shi and S. Ruan, "Spatial, temporal and spatiotemporal patterns of diffusive predator-prey models with mutual interference," IMA Journal of Applied Mathematics, vol. 80, no. 5, pp. 1534-1568, 2015.

[14] Y. Song, H. Jiang, Q.-X. Liu, and Y. Yuan, "Spatiotemporal dynamics of the diffusive Mussel-Algae model near turing-hopf bifurcation," SIAM Journal on Applied Dynamical Systems, vol. 16, no. 4, pp. 2030-2062, 2017.

[15] Y. Song and X. Tang, "Stability, steady-state bifurcations, and turing patterns in a predator-prey model with herd behavior and prey-taxis," Studies in Applied Mathematics, vol. 139, no. 3, pp. 371-404, 2017.

[16] Y. Cai, Z. Gui, X. Zhang, H. Shi, and W. Wang, "Bifurcations and pattern formation in a predator-prey model," International Journal of Bifurcation and Chaos, vol. 28, no. 11, p. 1850140, 2018. 
[17] Y. Cai, X. Lian, Z. Peng, and W. Wang, "Spatiotemporal transmission dynamics for influenza disease in a heterogenous environment," Nonlinear Analysis: Real World Applications, vol. 46, pp. 178-194, 2019.

[18] W. Wang, X. Gao, Y. Cai, H. Shi, and S. Fu, "Turing patterns in a diffusive epidemic model with saturated infection force," Journal of The Franklin Institute, vol. 355, no. 15, pp. 7226-7245, 2018.

[19] F. Rao and Y. Kang, "The complex dynamics of a diffusive prey-predator model with an Allee effect in prey," Ecological Complexity, vol. 28, pp. 123-144, 2016.

[20] P.-P. Liu, "An analysis of a predator-prey model with both diffusion and migration," Mathematical and Computer Modelling, vol. 51, no. 9-10, pp. 1064-1070, 2010.

[21] W. M. Wang, Q. X. Liu, and J. Zhen, "Spatiotemporal complexity of a ratio-dependent predator-prey system," Physical Review E: Statistical, Nonlinear, and Soft Matter Physics, vol. 75, no. 5, pp. 051913-051921, 2007.

[22] H. Zhang, S. Ma, T. Huang, X. Cong, H. Yang, and F. Zhang, "A new finding on pattern self-organization along the route to chaos," Chaos, Solitons \& Fractals, vol. 106, pp. 118-130, 2018.

[23] D. C. Mistro, L. A. D. Rodrigues, and S. Petrovskii, "Spatiotemporal complexity of biological invasion in a space- and time-discrete predator-prey system with the strong Allee effect," Ecological Complexity, vol. 9, pp. 16-32, 2012.

[24] T. Huang, H. Zhang, H. Yang, N. Wang, and F. Zhang, "Complex patterns in a space- and time-discrete predator-prey model with beddington-deangelis functional response," Communications in Nonlinear Science and Numerical Simulation, vol. 43, pp. 182199, 2017.

[25] T. Huang and H. Zhang, "Bifurcation, chaos and pattern formation in a space- and time-discrete predator-prey system," Chaos, Solitons \& Fractals, vol. 91, pp. 92-107, 2016.

[26] T. Huang, H. Zhang, and H. Yang, "Spatiotemporal complexity of a discrete space-time predator-prey system with self- and cross-diffusion," Applied Mathematical Modelling: Simulation and Computation for Engineering and Environmental Systems, vol. 47, pp. 637-655, 2017.

[27] L. A. D. Rodrigues, D. C. Mistro, and S. Petrovskii, "Pattern formation in a space- and time-discrete predator-prey system with a strong Allee effect," Theoretical Ecology, vol. 5, no. 3, pp. 341-362, 2012.

[28] K. Kaneko, "Transition from torus to chaos accompanied by frequency lockings with symmetry breaking in connection with the coupled-logistic map," Progress of Theoretical and Experimental Physics, vol. 69, no. 5, pp. 1427-1442, 1983.

[29] K. Kaneko, "Period-doubling of kink-antikink patterns, quasiperiodicity in antiferro-like structures and spatial intermittency in coupled logistic lattice towards a prelude of a "field theory of chaos"," Progress of Theoretical and Experimental Physics, vol. 72, no. 3, pp. 480-486, 1984.

[30] K. Kaneko, "Spatiotemporal intermittency in coupled map lattices," Progress of Theoretical and Experimental Physics, vol. 74, no. 5, pp. 1033-1044, 1985.

[31] D. Punithan, D.-K. Kim, and R. I. McKay, "Spatio-temporal dynamics and quantification of daisyworld in two-dimensional coupled map lattices," Ecological Complexity, vol. 12, pp. 43-57, 2012.

[32] F. Zhang, H. Zhang, S. Ma, T. Meng, T. Huang, and H. Yang, "Self-organized patterns induced by Neimark-Sacker, flip and turing bifurcations in a discrete predator-prey model with
Lesie-Gower functional response," Entropy, vol. 19, no. 6, p. 258, 2017.

[33] W. Yang, Spatiotemporal Chaos and Coupled Map Lattice, Shanghai Science and Technology Education Press, Shanghai, China, 1994.

[34] N. Jonsson, B. Jonsson, J. Skurdal, and L. P. Hansen, "Differential response to water current in offspring of inlet-and outlet-spawning brown trout Salmo trutta," Journal of Fish Biology, vol. 45, no. 2, pp. 356-359, 1994.

[35] M. P. Hassell, O. Miramontes, P. Rohani, and R. M. May, "Appropriate formulations for dispersal in spatially structured models: comments on bascompte \& solé," Journal of Animal Ecology, vol. 64, no. 5, pp. 662-664, 1995.

[36] S. MacNamara and G. Strang, "Operator splitting," in Splitting Methods in Communication, Imaging, Science, and Engineering, Springer International Publishing, New York, NY, USA, 2016.

[37] J. M. Fryxell and A. R. E. Sinclair, "Causes and consequences of migration by large herbivores," Trends in Ecology \& Evolution, vol. 3, no. 9, pp. 237-241, 1988.

[38] M. G. Neubert, H. Caswell, and J. D. Murray, "Transient dynamics and pattern formation: reactivity is necessary for Turing instabilities," Mathematical Biosciences, vol. 175, no. 1, pp. 1-11, 2002.

[39] L. Bai and G. Zhang, "Nontrivial solutions for a nonlinear discrete elliptic equation with periodic boundary conditions," Applied Mathematics and Computation, vol. 210, no. 2, pp. 321333, 2009.

[40] J. van de Koppel, M. Rietkerk, N. Dankers, and P. M. J. Herman, "Scale-dependent feedback and regular spatial patterns in young mussel beds," The American Naturalist, vol. 165, no. 3, pp. E66-E77, 2005.

[41] C. Tian and L. Zhang, "Delay-driven irregular spatiotemporal patterns in a plankton system," Physical Review E: Statistical Nonlinear \& Soft Matter Physics, vol. 88, no. 1, Article ID 012713, 2013.

[42] R. K. Upadhyay, W. Wang, and N. K. Thakur, "Spatiotemporal dynamics in a spatial plankton, system," Mathematical Modelling of Natural Phenomena, vol. 5, no. 5, pp. 102-122, 2012.

[43] H. Malchow, "Non-equilibrium spatio-temporal patterns in models of non-linear plankton dynamics," Freshwater Biology, vol. 45, no. 2, pp. 239-251, 2000.

[44] A. B. Medvinsky, S. V. Petrovskii, I. A. Tikhonova, H. Malchow, and B. L. Li, "Spatiotemporal complexity of plankton and fish dynamics," SIAM Review, vol. 44, no. 3, pp. 311-370, 2002. 


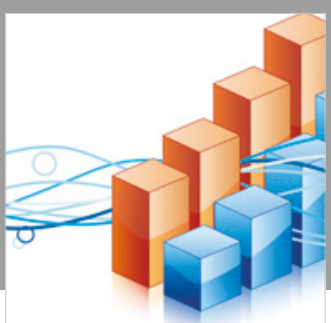

Advances in

Operations Research

\section{-n-m}
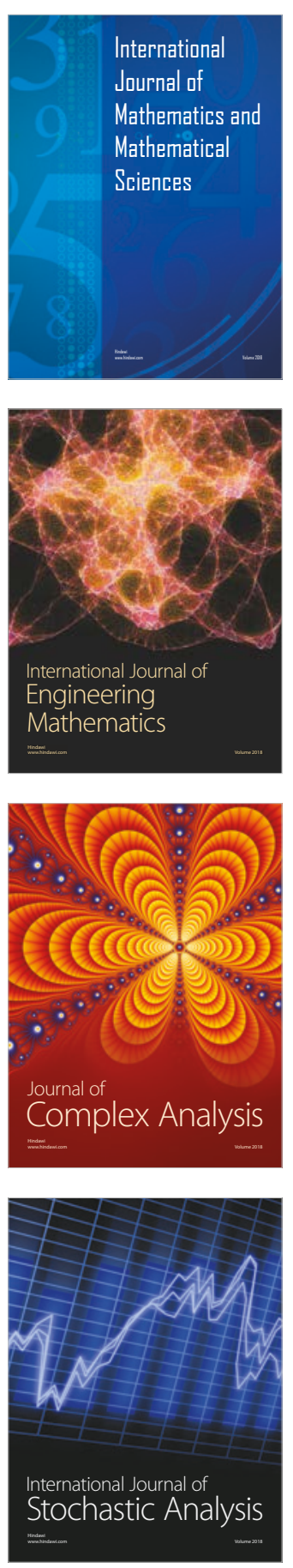
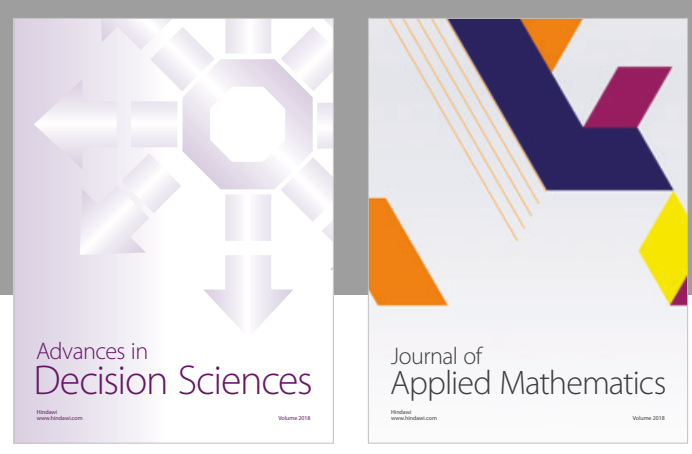

Journal of

Applied Mathematics
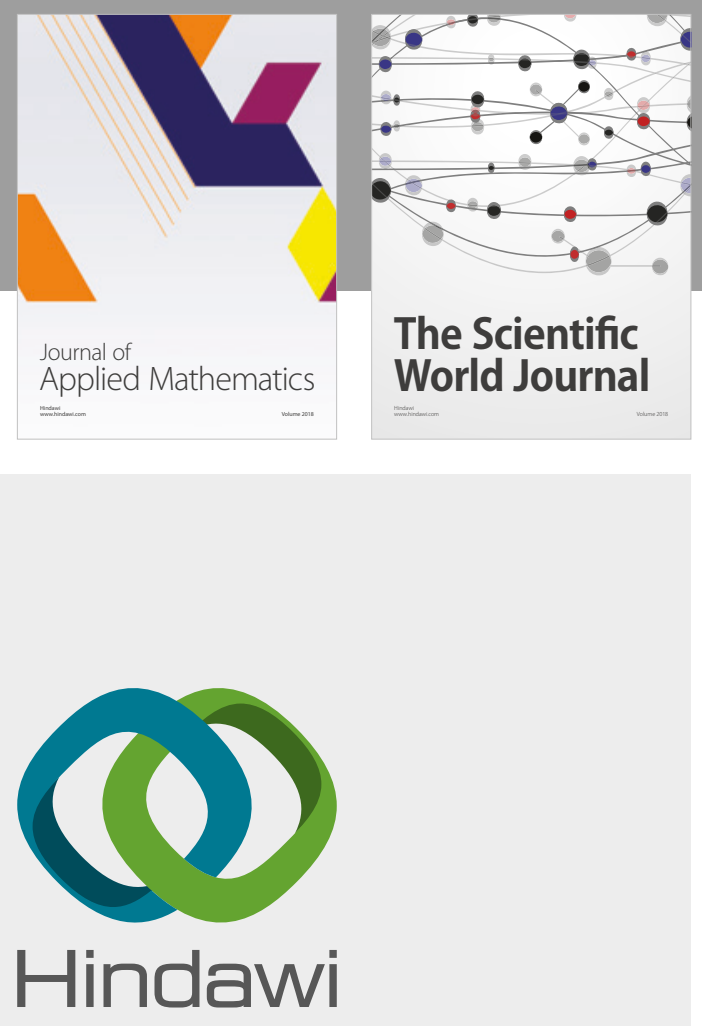

Submit your manuscripts at

www.hindawi.com

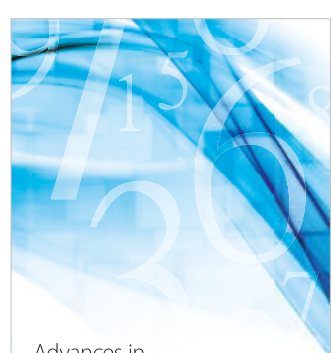

Advances in
Numerical Analysis
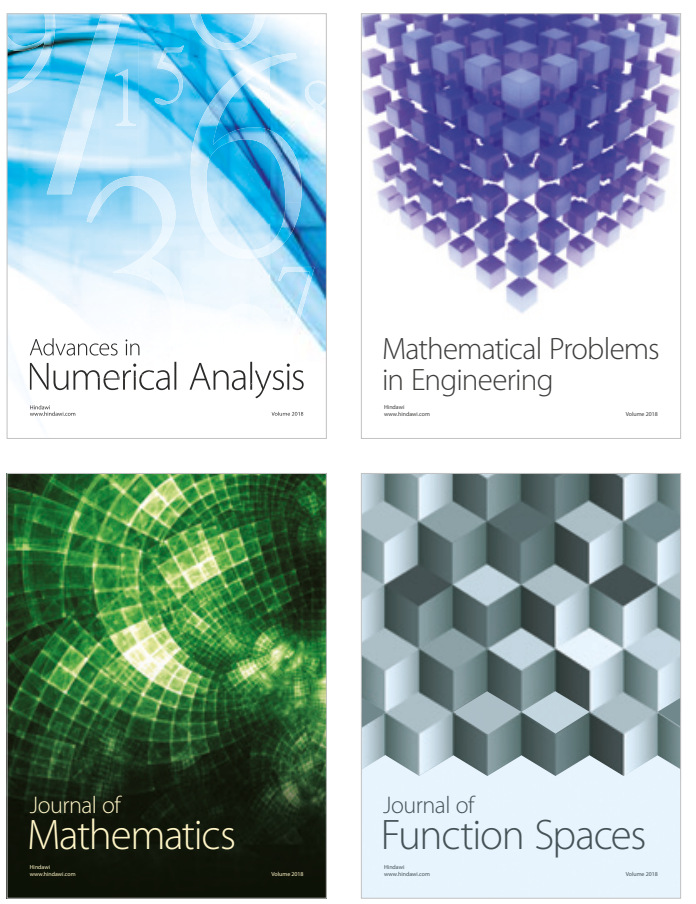

Mathematical Problems in Engineering

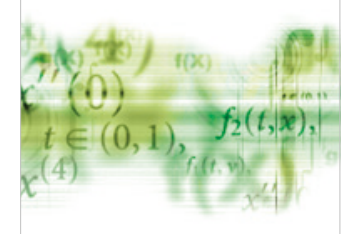

International Journal of

Differential Equations

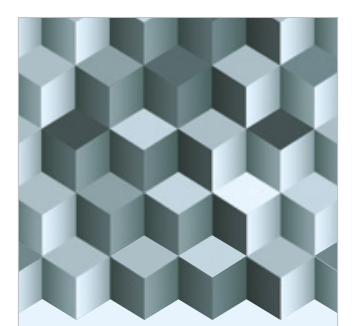

Journal of

Function Spaces
The Scientific

World Journal

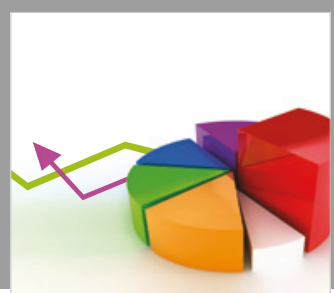

Journal of

Probability and Statistics
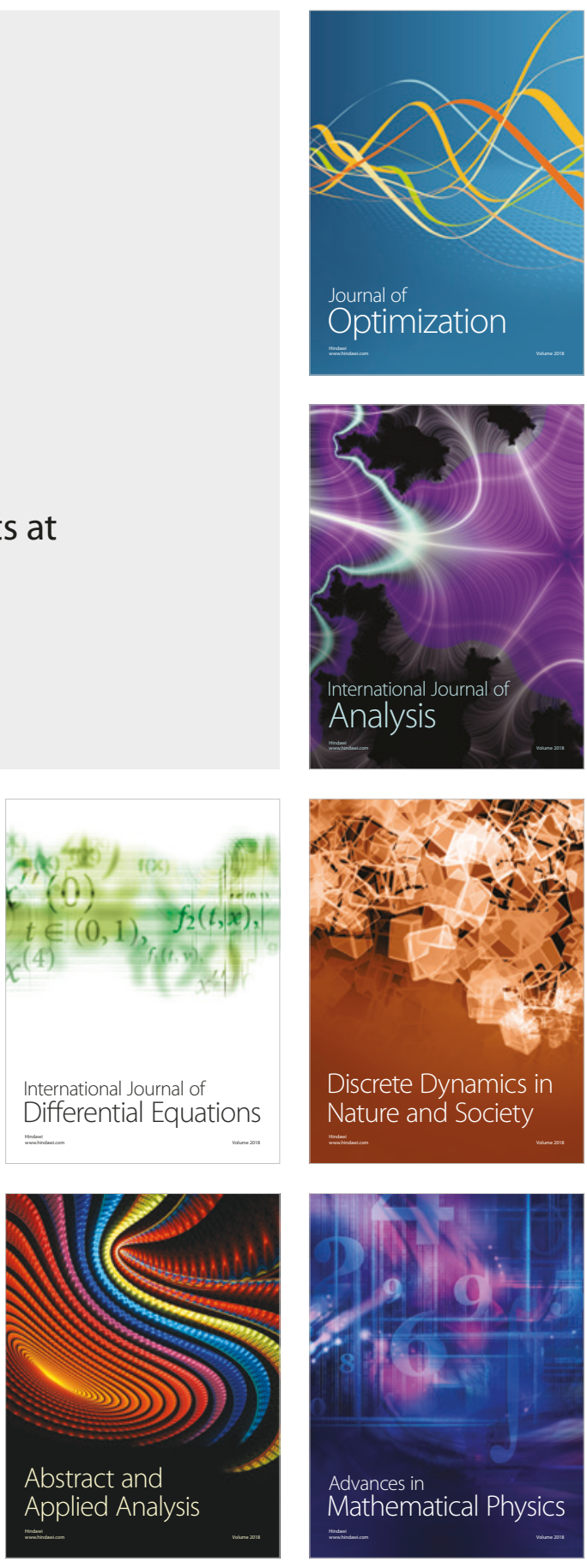